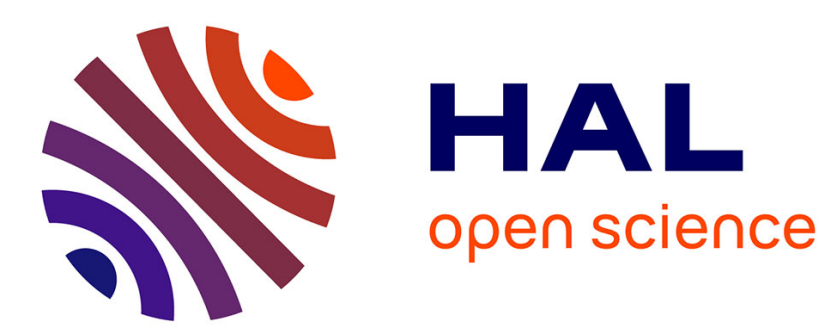

\title{
Experiments on Authorship Attribution by Intertextual Distance in English
}

Dominique Labbé

\section{To cite this version:}

Dominique Labbé. Experiments on Authorship Attribution by Intertextual Distance in English. Journal of Quantitative Linguistics, 2007, 14 (1), pp.33-80. halshs-00139070

\section{HAL Id: halshs-00139070 \\ https://shs.hal.science/halshs-00139070}

Submitted on 7 Mar 2008

HAL is a multi-disciplinary open access archive for the deposit and dissemination of scientific research documents, whether they are published or not. The documents may come from teaching and research institutions in France or abroad, or from public or private research centers.
L'archive ouverte pluridisciplinaire $\mathbf{H A L}$, est destinée au dépôt et à la diffusion de documents scientifiques de niveau recherche, publiés ou non, émanant des établissements d'enseignement et de recherche français ou étrangers, des laboratoires publics ou privés. 
Dominique Labbé

(dominique.labbe@iep-grenoble.fr)

CERAT-IEP

BP 48

F 38040 Grenoble Cedex 9

\section{Experiments on Authorship Attribution by Intertextual Distance in English}

Draft of the paper published in :

Journal of Quantitative Linguistics, 2007, Volume 14, Number 1, pp. 33-80.

\section{Résumé}

A quelles conditions peut-on affirmer que des textes sont "proches" ou "éloignés" les uns des autres ? Est-ce que des textes d'un même auteur sont plus similaires entre eux que ceux d'auteurs différents ? La réponse à ces questions est donnée par le calcul de la distance intertextuelle combinée à diverses méthodes de classification. Un test en aveugle et quelques expériences complémentaires montrent que cette méthode offre un outil intéressant pour résoudre les problèmes d'attribution d'auteur par des voies non-traditionnelles.

\footnotetext{
Abstract

How can it be said that texts are "near" or "distant" from one another? Are different texts by a single author more similar than texts by different authors? To answer these questions, a method is proposed by combination of the calculus of intertextual distance with automatic clustering and tree-classification. A blind test and some additional experiments show that this method offers an interesting tool for non-traditional authorship attribution.
} 


\section{Experiments on Authorship Attribution by Intertextual Distance in English}

How can it be said that texts are "near to" or "distant from" one another? Are different texts by a single author more similar than texts by different authors? These are two of the basic questions of authorship (On this question, see: Love, 2002 and for a discussion: Rudman, 1998). Rather than using a set of "frequent words" (Holmes, 1995, Burrows, $2002 \&$ 2003) or "collocations" (Hoover, 2001) or "function words" (Eliott \& Valenza, 2001), more or less arbitrarily selected, it would be better to make use of the entire vocabulary of the texts in order to measure proximity. Following this simple idea, a method is proposed to establish authorship by calculating "intertextual distance" (Labbé \& Labbé, 2001, 2003).

An index of intertextual distance measures the influence of four factors familiar to literary critics: subject (content, themes), genre, epoch (chronology) and author. A great number of experiments with French texts led to the conclusion that, when looking at texts written during the same period, in the same genre, the predominant factor is nearly always authorship (this tendency seems to be verified also on some English texts: Merriam, 2003b). Thus, this calculation offers an interesting tool for non-traditional authorship attribution.

Tom Merriam applied this method to Marlowe, Shakespeare and Middleton (Merriam, 2002b and 2003a) and some contemporaneous texts (Merriam, 2003b). The results proved encouraging. Thus a question was asked: "Could this technique be extended in English to other authors in the same way that it has been done in French?" Two different experiments gave a first answer to this question.

\section{A BLIND-TEST}

At the end of January 2004, a blind-test was begun on a corpus of 52 texts - each of 10,000 tokens in length, drawn from English novels - without any indication of the authors' identities or titles of the works (in the following this corpus is named "Oxquarry1"). Texts were supplied by Gerard Ledger who was himself concerned by this question (Ledger, 1995). He chose samples from more or less contemporaneous novels, the authorship of which he thought was difficult to establish by means of their vocabularies. The samples were based on 
texts downloaded from the Gutenberg Project. All texts were checked for errors and edited so as to be easily machine readable. However they were not checked against the original works, and only obvious errors were removed or corrected (nevertheless it may be assumed that they are accurate, in that the Gutenberg Project texts are mostly of a high standard). Each excerpt was coded with an alpha-numeric tag in the series $1 \mathrm{~A}-1 \mathrm{Z}$ followed by $2 \mathrm{~A}-2 \mathrm{Z}$, giving 52 excerpts. In this article these codes are used throughout, but Appendix 1 discloses authors, titles and chapters of the novels from which these excerpts were drawn. This information was supplied by Dr. Ledger after the following text was sent to him (actual authors and titles are added in order to facilitate the reading of some of the tables and charts).

The question asked was not: "Who wrote what?" but, more modestly: "Is it possible to know which texts are written by the same author and which ones are from different hands?"

To answer this question, a three-step method was used. First, the indices, measuring the distances separating the vocabulary of each pair of texts, were calculated. Secondly, a direct examination of these indices brought out the values which indicate identity of authorship. Thirdly, this separation was complemented by further classifications.

\section{Preliminary remark.}

All samples were processed with software written by C. \& D. Labbé. The first stage had been designed in parallel with the well-known Oxford Concordance Program (Hockey and Martin, 1988): it lists the words used in the text and calculates their frequencies (the two programs give exactly the same results). Then it calculates the inter-textual distances.

\subsection{Calculation of inter-textual distance}

Given two texts $A$ and $B$.

$-N_{a}$ and $N_{b}$ sizes of $A$ and $B$ in tokens;

$-F_{i a}$ and $F_{i b}$ the absolute frequencies of type $i$ in texts $A$ and $B$.

If their lengths (in tokens) are equal — as in this experiment - the distance index can be directly performed by subtracting the two frequencies of each type and by summing the results :

$$
D_{(A, B)}=\sum_{\mathrm{i} \in(\mathrm{A}, \mathrm{B})}\left|\mathrm{F}_{\mathrm{ia}}-\mathrm{F}_{\mathrm{ib}}\right| \text { with } N_{a}=N_{b}
$$


and the relative distance index is equal to:

$$
\text { (1) } \operatorname{Drel}_{(A, B)}=\frac{\sum_{i \in(A, B)}\left|F_{i a}-F_{i b}\right|}{2 N_{a}}
$$

$D_{(A, B)}$ gives the exact number of different words which separates the two texts. Relative distance index is expressed by a figure between 0 and 1 . It could also be defined as "the number of different tokens in an excerpt of 10,000 tokens" and expressed with integers.

This sum is an Euclidian "distance" which gives a quantitative answer to our opening question. Properties of Euclidian distances — identity, symmetry, triangle inequality — allow many useful operations to be performed, especially unbiased classifications (for the properties of textual distance index, see Labbé \& Labbé, 2001; Labbé \& Labbé, 2003 and below).

It should be noted that this index is given without estimate of uncertainty or error (of course, the spellings of words must have been checked).

The Corpus "Oxquarry1" is 520,000 tokens long. It is sufficient for a first experiment, even if it is not enough to give a full answer to all the questions about authorship attribution. Formula (1) was applied to this corpus. The calculation of all indices leads to a matrix of 52 lines * 52 columns, too large to be printed here. It contains 1,326 different indices (given the identity property, the diagonal matrix is null and given the symmetry property the upper half of the matrix is equal to the lower one).

A direct examination of this matrix gives an initial answer to the authorship question.

\subsection{Direct analysis of distance matrix}

G. Ledger supplied merely three pieces of information about the corpus Oxquarry1: 1) there were several authors; 2) there was a minimum of two excerpts by each author though not necessarily drawn from the same book; 3) all these texts belonged approximately to the same genre and the same period of time.

Thus if our preliminary assumption — giving authorship a predominant weight as opposed to content - is right, a simple prediction can be made: compared to a random distribution, the distance indices between pairs of excerpts by the same author should fall at the bottom of the distribution, and the highest part of this distribution should contain only pairs of texts by different authors. 
The mean of all indices is .4067 and the standard deviation $(\sigma)$ is equal to .0377 . With an uncertainty margin of $5 \%$, we can use two "milestones" (i.e. points on a continuum) to mark out significant values $( \pm 1.96 \sigma$ around the mean $)$ :

- lower limit (under which the authorship of the two excerpts is probably the same): $.4067-\left(1.96^{*} .0377\right)=.3328($ rounded to .333$)$.

- upper limit (above which the authorship of the two texts is probably different): $.4067+(1.96 * .0377)=.4806($ rounded to .481$)$.

\section{a. Same authorship (indices under .333)}

70 pairs of texts are under the lower margin (see appendix 2.1). For example, the first four lines of this table indicate that the distance between the nearest couples of texts are:

1D - 2W: .259

$1 \mathrm{~N}-2 \mathrm{E}: .265$

$1 \mathrm{~F}-2 \mathrm{E}: .267$

$1 \mathrm{~K}-2 \mathrm{~W}: .277$

Given the transitiveness of the inter-textual distance index, it can be said that since:

— texts $1 \mathrm{D}$ and $2 \mathrm{~W}$ are supposed by the same author;

— texts $1 \mathrm{~K}$ and $2 \mathrm{~W}$ are also supposed by the same hand;

these two relations imply that the texts $1 \mathrm{D}$ and $1 \mathrm{~K}$ may also be supposed by this same author...

In the same way, it can be said that since pairs of texts $1 \mathrm{~N}-2 \mathrm{E}$ and $1 \mathrm{~F}-2 \mathrm{E}$ are probably by the same author, similarly texts $1 \mathrm{~N}$ and $1 \mathrm{~F}$ may also be supposed by their same author, etc. (in fact, Appendix 1 shows that these excerpts are all by Stevenson).

These relations lead to 12 sets of texts of whose identical authorship is highly probable: this result is arrived at with greater than $95 \%$ certainty (Table 1). 
Table 1.12 text sets probably of same authorship

\begin{tabular}{|c|l|l|}
\hline 1 & $1 \mathrm{D}, 2 \mathrm{~W}, 1 \mathrm{~K}, 2 \mathrm{R}, 1 \mathrm{~N}, 2 \mathrm{E}, 1 \mathrm{~F}$ & Stevenson \\
\hline 2 & $1 \mathrm{~S}, 2 \mathrm{M}, 2 \mathrm{I}, 1 \mathrm{C}$ & Morris \\
\hline 3 & $1 \mathrm{E}, 1 \mathrm{Q}, 1 \mathrm{~B}, 2 \mathrm{~A}$ & Butler \\
\hline 4 & $2 \mathrm{~L}, 2 \mathrm{~T}, 2 \mathrm{~J}, 2 \mathrm{X}, 1 \mathrm{~L}, 2 \mathrm{D}, 1 \mathrm{X}, 2 \mathrm{~F}$ & Hardy \\
\hline 5 & $1 \mathrm{H}, 2 \mathrm{Z}, 2 \mathrm{~S}$ & Hardy \\
\hline 6 & $2 \mathrm{O}, 2 \mathrm{P}, 2 \mathrm{~V}$ & Forster \\
\hline 7 & $1 \mathrm{Z}, 2 \mathrm{C}, 1 \mathrm{Y}$ & Tressel \\
\hline 8 & $1 \mathrm{P}, 2 \mathrm{U}, 1 \mathrm{R}$ & Chesterton \\
\hline 9 & $2 \mathrm{H}, 1 \mathrm{G}, 1 \mathrm{O}$ & Conrad \\
\hline 10 & $2 \mathrm{~N}, 2 \mathrm{Q}, 2 \mathrm{~K}$ & Conrad \\
\hline 11 & $1 \mathrm{M}, 2 \mathrm{Y}, 1 \mathrm{~W}, 2 \mathrm{G}$ & Orczy \\
\hline 12 & $1 \mathrm{~J}, 2 \mathrm{~B}$ & Morris \\
\hline
\end{tabular}

At the end of this first step, five texts remain un-classified: 1A, 1I, 1T, $1 \mathrm{U}, 1 \mathrm{~V}^{1}$. It must be remembered that the goal was not so much to give a full answer as to avoid a false one.

\section{b. Different authorship (indices above .481)}

26 distance indices are above this upper margin (appendix 2.4). These pairs of texts are most probably not by the same author. For example: authorships of $1 \mathrm{~T}$ and $1 \mathrm{Z}$ are different as those of $1 \mathrm{~T}$ and $2 \mathrm{R}$. Given the fact that:

- $1 \mathrm{Z}$ belongs to Set 7, therefore $1 \mathrm{~T}$ cannot belong to this set 7 ;

$-2 \mathrm{R}$ belongs to Set 1 , therefore $1 \mathrm{~T}$ cannot belong to set 1 ;

For example, all these mutual exclusions lead to the following conclusions (about the five texts which remained un-classified after examination of the lower limit):

- $1 \mathrm{~T}$ can only belong to sets $4,5,8,9,10,11$;

- $1 \mathrm{~V}$ cannot belong to sets 1 and 12;

— the author of set 10 is different from the authors of sets 1,2 and 12;

It is worth noting that these negative conclusions are important because, in some cases, it is as valuable to be able to exclude a supposed authorship as to establish an unknown one. Of course, all these deductions are possible, taking into account the information given concerning the corpus (same genre, several authors and, at least, two texts for each author).

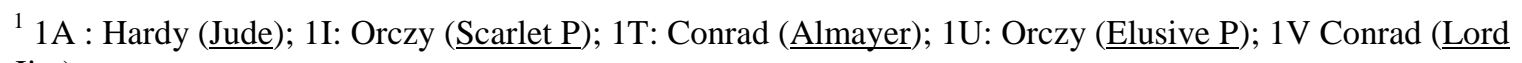
$\underline{\text { Jim). }}$
} 
When a large number of experiments are carried out, we are enabled to establish a standardised distance scale. Our paper (Labbé \& Labbé, 2001) presents such a scale for French texts. For example, if, in all experiments to be made on English texts, we never find a distance index under .34 between texts by two different authors, we will be able to assume the same authorship for any two texts when their inter-textual distance is below this threshold (for a 10,000 tokens standardised length).

In order to verify these conclusions and to propose some solutions for unsolved cases, two classification techniques are used.

\subsection{Automatic clustering}

The Dendrogram below (Fig. 1) gives a pictorial summary of this established analysis.

The algorithm starts by merging the two nearest excerpts (placed at the extreme left of the chart), and then recalculates the distance indices separating this new set from all other texts in order to calculate the next grouping. The nearest text is then placed next to the first two, and the procedure repeated until all texts have been placed in their correct position on the X-axis. Intertextual distance values corresponding to grouping stages are placed on the Y-axis.

The origin of Y-axis is placed at .25 in order to create a kind of "zoom effect" which facilitates reading the diagram, but it must not be forgotten that all the texts are relatively near to another...

When two texts $A, B$ are joined $(A \cup B)$, the properties of intertextual distance allow the calculation, without bias, of a new distance between this group and any text $(C)$ by the arithmetic mean of the original distance indices:

$$
\text { (2) } D_{(A \cup B, C)}=\frac{D_{(A, C)}+D_{(B, C)}}{2}
$$

The calculation is made by merging the two texts $A$ and $B$ and then by calculating the distance index separating this new "text" from $C$. For example, one can see on the extreme left of the dendrogram, that the horizontal edge between 1D and $2 \mathrm{~W}$ is placed just under .26 and that this group joins $1 \mathrm{~K}$ at a level of .275 (mean between indices $1 \mathrm{D}-1 \mathrm{~K}$ and $2 \mathrm{~W}-1 \mathrm{~K})^{2}$. Thus, horizontal lines which join vertical edges indicate the level of grouping. The higher this level, the more heterogeneous is the group, the more uncertain is the authorship.

\footnotetext{
${ }^{2}$ These three excerpts come from Catrionae by Stevenson (Appendix 1).
} 
Fig. 1. Cluster analysis on Corpus Oxquarry1

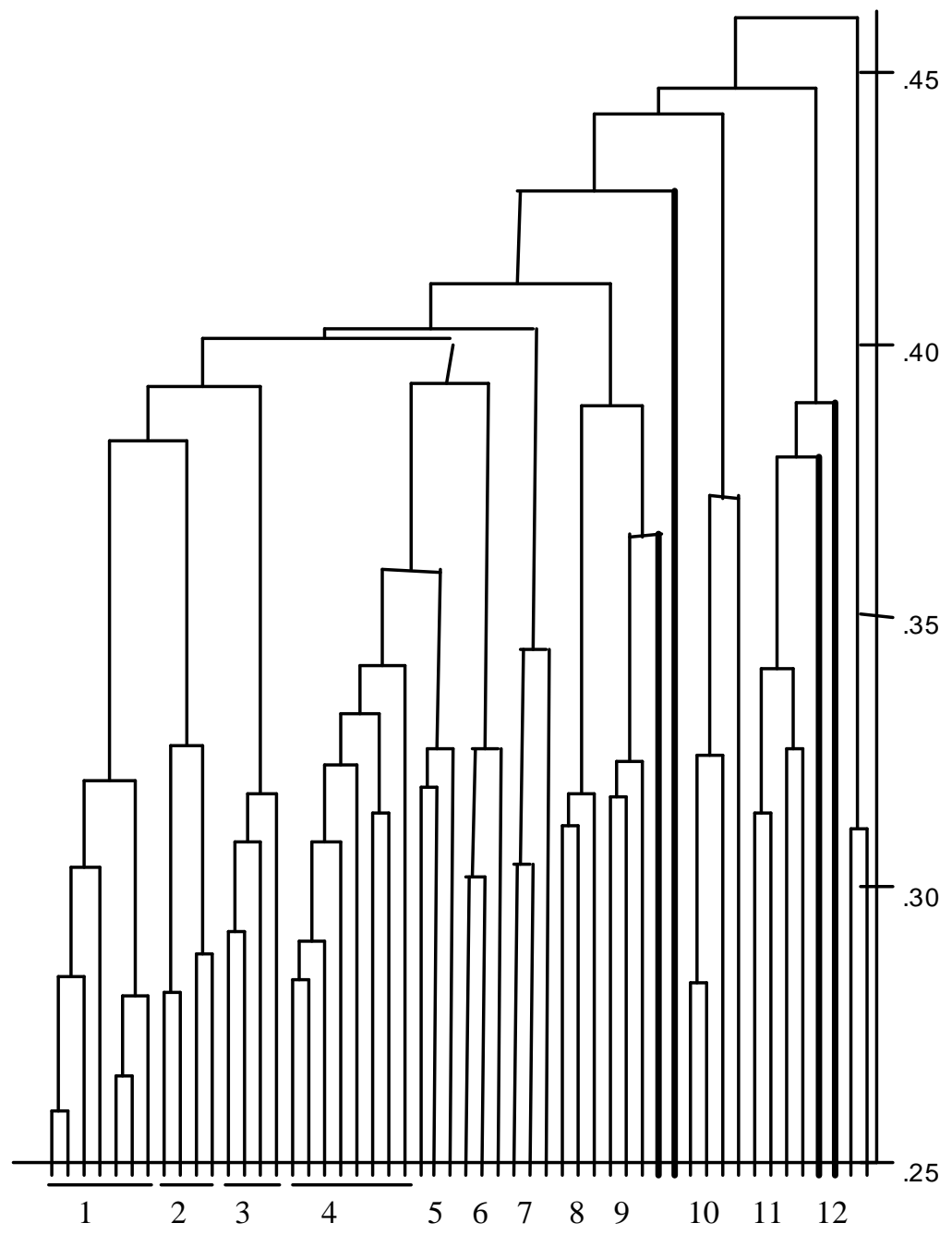

From left to rigth :

Set $1: 1 \mathrm{D}$ $2 \mathrm{~W}$

$1 \mathrm{~K}$

$2 \mathrm{R}$

$1 \mathrm{~N}$

$2 \mathrm{E}$

$1 \mathrm{~F}$

Set $2: 1 \mathrm{~S}$

$2 \mathrm{M}$

$1 \mathrm{C}$

2I

Set $3: 1 \mathrm{E}$

$1 \mathrm{Q}$

$1 \mathrm{~B}$

$2 \mathrm{~A}$
Set $4: 2 \mathrm{~L}$ $2 \mathrm{~T}$ 2J 2X 1X

1L 2D $2 \mathrm{~F}$ Set $5: 1 \mathrm{H}$ $2 Z$ $2 \mathrm{~S}$ Set $6: 20$ $2 \mathrm{P}$ $2 \mathrm{~V}$ Set $7: 1 \mathrm{Z}$ 
Cluster analysis confirms the conclusions presented above and drawn from the direct examination of the inter-textual distance matrix. It can be seen that all sets have their primary nodes under .35 (calculated by automatic clustering using formula 2). This value is likely to be a threshold for identity of authorship.

In addition, some other conclusions appear to be highly probable (and congruent with the conclusions of direct inspection):

— text $1 \mathrm{~V}$ may be joined to set 9 (the node is slightly over .35);

— set 4 may be joined to set 5 : here again, the node is slightly over .35 .

Two other solutions are also suggested and they are congruent with the conclusions of direct inspection ${ }^{3}$ :

— text $1 \mathrm{~T}$ seems to be near set 10 but this node is far above . 35 ; thus it is impossible to choose between two equivalent solutions: same author, different theme (and/or different book and/or different date) or two different contemporaneous authors and the same theme;

— texts $1 \mathrm{U}$ and 1I seem to join set 11 (same uncertainty as with 1T);

In addition, $1 \mathrm{~A}$ is clearly too far from all the sets and cannot be classified.

A more complete answer is given with the help of a more powerful tool: tree-analysis.

\subsection{Tree-classification}

The trees below (Figs. 2 and 3) are drawn following Luong's formulae: "valued" trees and "grouping" method (Luong, 1988). These formulae, methods and algorithms are fully explained in Rulhman (2003) and in Labbé \& Labbé (2005).

Firstly, this analysis was applied to the original matrix (Fig. 2).

Each "vertice" or terminal "leaf" is tagged with its Corpus code. For example, $1 \mathrm{~W}$ and 2G (top of the tree), are adjacent, as also are 1B and 1E. They form two sets of "neighbours" and these two groups are opposed. The edges ("stems" or "branches") link those four leafs to two nodes which are created by the algorithm. Their relative positions are calculated in order to create edges proportional to the original distances. A leaf of the tree is linked to another by a path formed by, at least, one edge. The longer the path, the farther apart are the two texts.

This graph is "valued". That is to say that the path lengths are positive and in fact proportional to the original values in the corresponding cells of the distance matrix. This

\footnotetext{
${ }^{3}$ In fact, these four solutions are correct: $1 \mathrm{~V}, 1 \mathrm{U}$, sets 9 and 11 are by Orczy; Hardy is the author of Set 4 and 5; Conrad is the one of $1 \mathrm{~T}$ and set 10 (Appendix 1).
} 
calculation is very complex because this tree must represent, as exactly as possible, the lengths of 1,326 different links.

Fig. 2 Tree-classification on "raw" distance indices

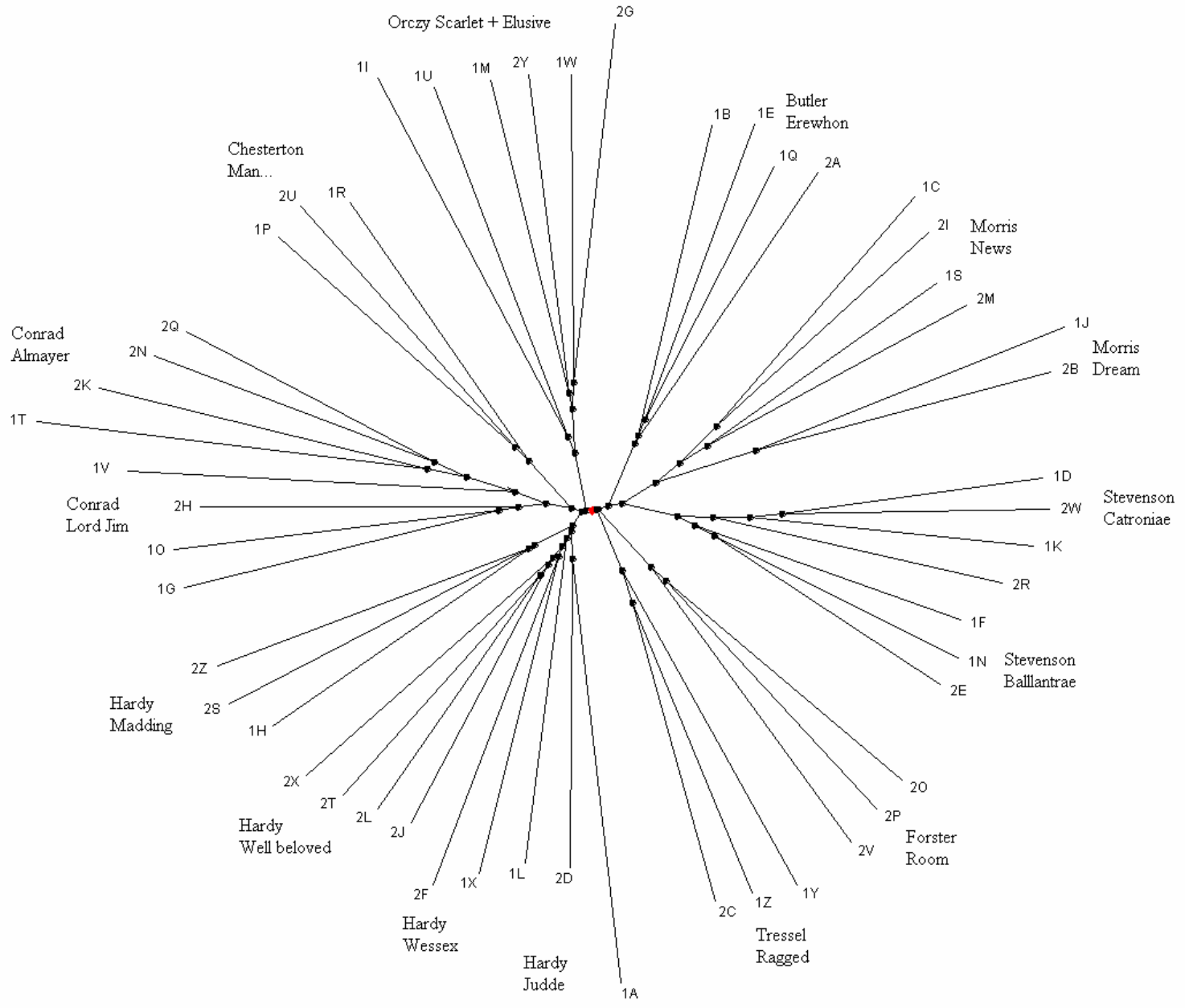

Among various innovations, a measure of quality is proposed. The quality of a tree, such as that presented in Fig. 2, can be evaluated by comparing the 1,326 original indices with all corresponding path lengths on the tree. If all these paths are exactly equal to their corresponding distance indices, the quality index will be equal to 1 . This index is calculated for each path, for each node and for the whole tree. Above .95 , the tree can be considered as an excellent representation of similarities and dissimilarities existing between all the texts. Here the quality index is equal to .975 . In other words, $97.5 \%$ of the whole information contained in the distance matrix is faithfully represented on this tree. For the 51 nodes, only one index is equal to .934 ; all other indices are over .95 . All path quality indices are over .90 . 
A high degree of confidence can be given to this chart. In all its parts, this tree gives credible information.

Firstly one notices the cross shape (or star shape) around the tree root: a small grey lozenge (or red one in electronic files) located exactly at the tree "centre". Terminal edges are very long and nodes, black dots, are often very near the centre of the tree. This shape means that all these texts are roughly "equidistant" except a few pairs - like 1D-2W, $1 \mathrm{~W}-2 \mathrm{G}$ (the first nodes are relatively far from the central trunk) - or some texts standing apart (1A, 1T)...

This shape of tree makes it difficult to observe slight differences between texts. Thus the tree can be "reduced" in order to obtain a kind of "zoom effect" on the central trunk of the diagram and to reduce proportionally the length of terminal leaves (in Fig. 3, original distance indices have been "reduced" by 0.2 ). In other words, this reduction highlights the "contrast" between texts which are actually very near to one another. Of course this "reduction" entails some disadvantages, as it lowers the quality of the tree.

Fig. 3 Tree-classification on distance indices less 0.2

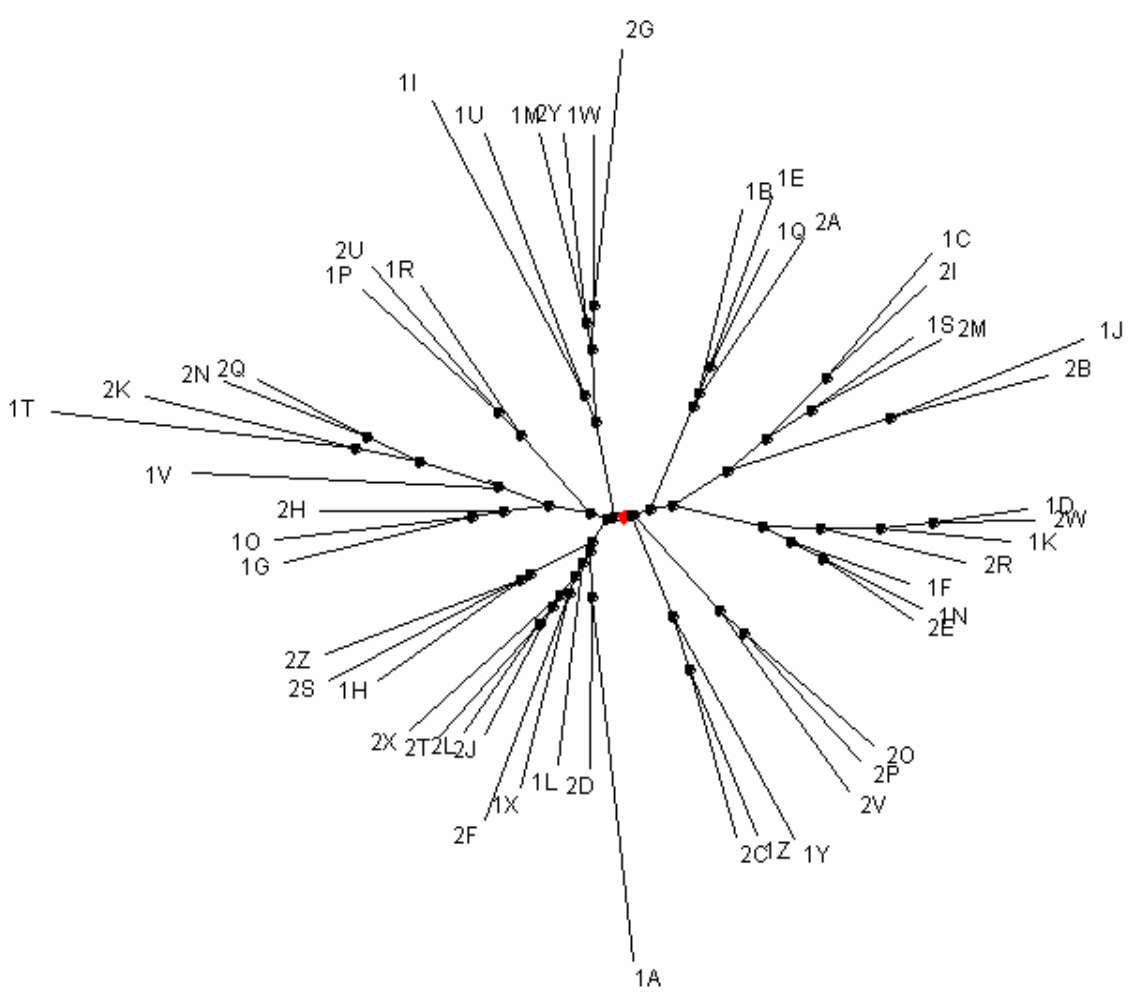

All the conclusions presented above are confirmed and a solution is proposed for the few remaining uncertainties. In addition, tree-classification suggests the reduction of the 12 sets (Table 1) to 9 by using the last junctions before the central trunk (Table 2 below). Texts 1I 
and $1 \mathrm{U}$ are aggregated to set 1 ; sets 2 and 12 seem to be by the same author; sets 4 and 5 and text $1 \mathrm{~A}$ are also assumed to be by one hand, as are sets 9,10 and texts 1T and 1V (clockwise on Fig. 3).

As G. Ledger confirmed: this classification is correct (in Table 2, author's names have been added in order to facilitate the reading).

Table 2. The nine text sets of probable same authorship. Clockwise on Fig. 2 and 3 (sets already established before tree-classification are placed between brackets)

\begin{tabular}{|c|c|l|l|}
\hline Final sets & Table 1 & Excepts (between brackets : Table 1) & Authors \\
\hline 1 & 11 & $1 \mathrm{I}, 1 \mathrm{U}+\{1 \mathrm{M}, 2 \mathrm{Y}, 1 \mathrm{~W}, 2 \mathrm{G}\}$ & Orczy \\
\hline 2 & 3 & $\{1 \mathrm{~B}, 1 \mathrm{E}, 1 \mathrm{Q}, 2 \mathrm{~A}\}$ & Butler \\
\hline 3 & $2+12$ & $\{1 \mathrm{C}, 2 \mathrm{I}, 1 \mathrm{~S}, 2 \mathrm{M}\}+\{1 \mathrm{~J}, 2 \mathrm{~B}\}$ & Morris \\
\hline 4 & 1 & $\{1 \mathrm{D}, 2 \mathrm{~W}, 1 \mathrm{~K}, 2 \mathrm{R}, 1 \mathrm{~F}, 1 \mathrm{~N}, 2 \mathrm{E}\}$ & Stevenson \\
\hline 5 & 6 & $\{2 \mathrm{O}, 2 \mathrm{P}, 2 \mathrm{~V}\}$ & Foster \\
\hline 6 & 7 & $\{1 \mathrm{Z}, 2 \mathrm{C}, 1 \mathrm{Y}\}$ & Tressel \\
\hline 7 & $4+5$ & $1 \mathrm{~A}+\{2 \mathrm{D}, 1 \mathrm{~L}, 1 \mathrm{X}, 2 \mathrm{~L}, 2 \mathrm{~F}, 2 \mathrm{~J}, 2 \mathrm{~T}, 2 \mathrm{X}\}+\{1 \mathrm{H}, 2 \mathrm{~S}, 2 \mathrm{Z}\}$ & Hardy \\
\hline 8 & $9+10$ & $\{1 \mathrm{G}, 1 \mathrm{O}, 2 \mathrm{H}\}+1 \mathrm{~V}+\{1 \mathrm{~T}, 2 \mathrm{~K}, 2 \mathrm{~N}, 2 \mathrm{Q}\}$ & Conrad \\
\hline 9 & 8 & $1 \mathrm{P}, 2 \mathrm{U}, 1 \mathrm{R}$ & Chesterton \\
\hline
\end{tabular}

\subsection{Some remarks about this test}

\section{What degree of certainty can be assigned to these conclusions?}

The sets placed between brackets in table 2 are the only "certain" authorships. In addition, the grouping of the two sets of excerpts by Hardy can be considered as highly probable, because it is clearly indicated by automatic clustering, as well as by tree classification. Other set groupings are "the best fit ones" — taking into account the fact that each author has at least two texts - among all solutions possible, but one cannot assign them a numerical probability. Lengths on the dendrogram, or the tree, give an approximate answer. For example, it can be seen that sets of excerpts by Hardy join together just before reaching the centre of the tree. In Fig. 2, the length of the last edge- joining these sets to the central trunk - is very short: less than $10 \%$ of the mean length of the terminal edges of these groups. Of course, the algorithm of classification is accurate but, when differences are so small... it is difficult to choose between two solutions: (1) same author and different themes and/or books; 
(2) two contemporaneous authors and a same theme? The same remark applies to pairs $\{1 \mathrm{~T}$ $2 \mathrm{~K}\}$ and $\{1 \mathrm{I}-1 \mathrm{U}\}$.

If both cluster and tree classifications have not the same validity as a direct examination of the matrix (with the help of a normalised scale that is yet to be created), their results converge clearly toward the same conclusions. Cluster analysis and tree analysis are therefore reliable tools for literary studies. Further experiments should lead to the calibration of a normalised scale for authorship attribution, like the one used on French texts by Labbé \& Labbé (2001 \& 2003), which give a standardised degree of assurance.

\section{Is success by chance alone possible?}

If there were only pairs of texts, what is the probability of finding all the 26 correct answers by chance? Given the fact that there are 1,326 possible different couples of texts, the probability of success by chance is:

$$
\mathrm{P}=26 / 1,326 * 25 / 1,325 *(\ldots) * 2 / 1,301 * 1 / 1,300
$$

This probability is almost infinitesimal. And this figure must be reduced to accommodate possible trios, quartets, etc. Who can easily imagine the number of different combinations that can be made with only 52 objects? ${ }^{4}$

The trial was potentially a difficult one, although the organiser wrote after the trial:

"I ought to point out a concern, which is that critics may object that the texts are relatively easy to differentiate. Some of them should separate into groups quite readily because of certain idiosyncracies, for example the Scottish dialect in the Stevenson work; or the working class dialogue in the Tressel work."

In reply, it is to be noted that, if these idiosyncracies are likely to be prominent details for a human reader, they weigh very little in the whole text surface and in the calculation of the inter-textual distance index. Above all, distances between texts stem from "common" words which are likely to be found in every writer.

Which words? It is an important question to which an answer can be made using Corpus Oxquarry1.

\footnotetext{
${ }^{4}$ Here ends the text sent, in February 2004, to G. Ledger in order to present him the results of the "blind-test".
} 


\subsection{Contribution of types (vocabulary words) to the inter-textual distances}

Where do the distances between texts come from? Or more precisely, two questions may be asked with the help of Corpus Oxquarry1.

First question: Do the differences between texts stem from rare words or from frequent ones?

To answer this question, the software programme calculates, for each pair of texts (A, $B)$, the distance indices between each pair of the same word types which belong to the union of the two texts $(A U B)$. In the case of the type $i(i \in A, B)$, the "absolute contribution to the distance" (between texts $A$ and $B$ ) for type $i$ is equal to:

$$
D\left(i_{a}, i_{b}\right)=\left|F_{i a}-F_{i b}\right|
$$

The surface area of the texts compared in this computation is equal to $F_{i a}+F_{i b}$.

From these can be extracted the "distance index" or "relative distance" for the word type $i$ :

$$
\operatorname{Drel}\left(i_{a}, i_{b}\right)=\frac{\left|F_{i a}-F_{i b}\right|}{F_{i a}+F_{i b}}
$$

The "types" may be then ranked by ascending frequency. The contribution of each relative frequency group is calculated by summing up the absolute contributions (column $A$ in the table 3 below). This allows one to measure the contribution of each group or frequency class to the greater or lesser distancing between the two texts under consideration $(A \%)$, as well as the text surface covered by this class (Columns $B$ and $B \%$ ), and the relative distance (Column C).

Does the value of $C$ differ significantly or not from the average for all the word types? For all of Corpus Oxquarry1, the average or mean distance index is 0.406 (AvgDrel) and the standard deviation $(\sigma)$ is equal to 0.0377 . The $z$-value, or standardised value of the random variable (column $D$ ) is equal to:

$$
\frac{\operatorname{Drel}\left(i_{a}, i_{b}\right)-\operatorname{Avg}(\text { Drel })}{\sigma}
$$


Table 3: Contribution by grouped frequency class to the distances (Corpus Oxquarry1)

\begin{tabular}{|c|c|c|c|c|c|c|}
\hline $\begin{array}{l}\text { Frequency } \\
\text { Class }\end{array}$ & $\begin{array}{c}\text { A } \\
\text { Absolute } \\
\text { Distance }\end{array}$ & $\begin{array}{c}\mathrm{A} \% \\
\% \text { of Total } \\
\text { Distance }\end{array}$ & $\begin{array}{c}\text { B } \\
\text { Theoretical } \\
\text { Surface Text }\end{array}$ & $\begin{array}{c}\mathrm{B} \% \\
\% \text { of Total } \\
\text { Theoretical } \\
\text { Text }\end{array}$ & $\begin{array}{c}\mathrm{C}=\mathrm{A} / \mathrm{B} \\
\text { Distance } \\
\text { Index }\end{array}$ & $\begin{array}{c}\mathrm{D} \\
Z \text {-value }\end{array}$ \\
\hline 1 to 4 & $10,104,730$ & 47.0 & $12,105,150$ & 22.8 & 0.835 & 16.3 \\
\hline 5 to 9 & $2,553,474$ & 11.9 & $4,480,490$ & 8.4 & 0.570 & 6.3 \\
\hline 10 to 14 & $1,233,152$ & 5.7 & $2,639,652$ & 5.0 & 0.467 & 2.4 \\
\hline 15 to 19 & 815,530 & 3.8 & $1,986,830$ & 3.7 & 0.410 & 0.2 \\
\hline 20 to 29 & $1,098,062$ & 5.1 & $2,940,266$ & 5.5 & 0.373 & -1.2 \\
\hline 30 to 49 & $1,096,182$ & 5.1 & $3,426,642$ & 6.5 & 0.320 & -3.2 \\
\hline 50 to 99 & $1,340,886$ & 6.2 & $5,066,190$ & 9.6 & 0.265 & -5.3 \\
\hline 100 to 199 & $1,202,394$ & 5.6 & $5,335,286$ & 10.1 & 0.225 & -6.8 \\
\hline 200 to 499 & $1,242,266$ & 5.8 & $7,132,326$ & 13.4 & 0.174 & -8.8 \\
\hline 500 to 999 & 588,360 & 2.7 & $5,066,960$ & 9.6 & 0.116 & -11.0 \\
\hline$>999$ & 220,334 & 1.0 & $2,853,986$ & 5.4 & 0.077 & -12.5 \\
\hline Sum & $21,495,370$ & 100.0 & $53,033,778$ & 100.0 & 0.406 & \\
\hline
\end{tabular}

Within the $z$-value interval $(-1.96$ et +1.96$)$, we cannot say that an observed value significantly differs from the overall mean of .406 for all the words in Corpus Oxquarry1. Above and below these two limits, however, there is less than 1 chance in 20 of erring in stating that the observed value differs materially from the overall mean value.

For example, the least frequent "types", those of between 1 and 4 occurrences, contribute $10,105,000$ words, that is to say, $47 \%$ of the total distance. At the same time, they cover only $22.8 \%$ of the total "surface". Therefore the relative distance or index is equal to 0.835 $(10,104,730 / 12,105,150)$. The $z$-value of +16.3 indicates that there is virtually no chance of this being the result of random variation; the "rare words" are those which create the greatest distances between the texts. By contrast, the various frequent words (>499) contribute only $3.7 \%$ to this distance, while they occupy some $15 \%$ of the surface.

In referring to the article which appeared in Corpus (Labbé \& Labbé, 2003, Table 4, p. 113), one will recognize that, even though the profile is the same as in the table above, the $z-$ values are less in the case of a similar corpus of French works. In effect, lemmatization in French reduces the weighting of low frequency words and proportionally increases that of the overall average and high frequency words.

These contrasts suggest some important comments: For example, most linguistic analyses - notably PCA and factor analysis - are concerned only with the most frequent "types", whereas it is manifestly the rare words which are the principal differentiators of authors. 
Second question : among the frequent word types, which ones do and which ones do not differentiate between the texts?

Here the concern is only with the most frequent "types". One already knows that on average their contribution is weak. NB: The list of these words is based on the entire Corpus Oxquarry1 (the first column of the two tables 4 and 5 below gives the frequency of these "types" for the entire corpus). The reasoning is the same as above. The "types" are ranked according to their ascending contribution (i.e., increasing distance index). Tables 4 and 5 show the top and bottom of the list.

Table 4: The most frequent "types" (or function words) which contribute least to the distance between texts.

\begin{tabular}{|l|r|r|r|r|c|}
\hline \multicolumn{1}{|c|}{ Type } & Frequency & Distance & Text Surface & Distance index & $Z$-value \\
\hline to & 13147 & 99366 & 1347726 & 0.074 & -12.60 \\
\hline the & 29654 & 231374 & 3036642 & 0.076 & -12.51 \\
\hline in & 8351 & 82456 & 856188 & 0.096 & -11.75 \\
\hline of & 15975 & 167632 & 1635468 & 0.103 & -11.51 \\
\hline that & 6805 & 74808 & 697272 & 0.107 & -11.33 \\
\hline and & 16441 & 181310 & 1684326 & 0.108 & -11.32 \\
\hline for & 4248 & 47272 & 435132 & 0.109 & -11.28 \\
\hline at & 3199 & 38500 & 327828 & 0.117 & -10.94 \\
\hline with & 4296 & 56604 & 439824 & 0.129 & -10.52 \\
\hline a & 12144 & 162178 & 1245114 & 0.130 & -10.46 \\
\hline out & 1287 & 17254 & 132090 & 0.131 & -10.44 \\
\hline was & 7352 & 100688 & 751944 & 0.134 & -10.32 \\
\hline on & 3126 & 43358 & 320586 & 0.135 & -10.27 \\
\hline there & 1841 & 27330 & 189414 & 0.144 & -9.92 \\
\hline as & 4448 & 67982 & 456246 & 0.149 & -9.74 \\
\hline not & 3486 & 55558 & 356286 & 0.156 & -9.48 \\
\hline from & 1863 & 29884 & 190332 & 0.157 & -9.44 \\
\hline this & 2263 & 36442 & 231846 & 0.157 & -9.43 \\
\hline if & 1630 & 26866 & 167382 & 0.161 & -9.31 \\
\hline what & 1597 & 26538 & 163914 & 0.162 & -9.25 \\
\hline but & 3387 & 56818 & 346902 & 0.164 & -9.18 \\
\hline
\end{tabular}


Table 5: The frequent "types" which contribute most to the distance between texts

\begin{tabular}{|l|c|r|r|r|c|}
\hline \multicolumn{1}{|c|}{ Type } & Frequency & Distance & Text Surface & Distance index & $Z$-value \\
\hline your & 977 & 40654 & 100266 & 0.4055 & 0.01 \\
\hline house & 474 & 20320 & 48552 & 0.4185 & 0.50 \\
\hline things & 350 & 15092 & 35904 & 0.4203 & 0.57 \\
\hline light & 300 & 13476 & 30804 & 0.4375 & 1.22 \\
\hline yes & 398 & 18172 & 41412 & 0.4388 & 1.27 \\
\hline moment & 320 & 14528 & 32640 & 0.4451 & 1.51 \\
\hline felt & 303 & 14350 & 30906 & 0.4643 & 2.24 \\
\hline my & 2529 & 122838 & 260406 & 0.4717 & 2.52 \\
\hline we & 1462 & 71168 & 149532 & 0.4759 & 2.69 \\
\hline rather & 385 & 19064 & 39168 & 0.4867 & 3.10 \\
\hline us & 511 & 25564 & 52224 & 0.4895 & 3.20 \\
\hline men & 592 & 29966 & 60690 & 0.4938 & 3.36 \\
\hline woman & 324 & 16430 & 33150 & 0.4956 & 3.43 \\
\hline our & 519 & 27104 & 53040 & 0.5110 & 4.02 \\
\hline don't & 497 & 26444 & 51204 & 0.5164 & 4.22 \\
\hline she & 3731 & 200024 & 380868 & 0.5252 & 4.56 \\
\hline her & 3977 & 214302 & 406062 & 0.5278 & 4.66 \\
\hline people & 456 & 25450 & 46614 & 0.5460 & 5.35 \\
\hline shall & 617 & 39172 & 63444 & 0.6174 & 8.06 \\
\hline work & 318 & 20596 & 32436 & 0.6350 & 8.73 \\
\hline sir & 312 & 22484 & 32436 & 0.6932 & 10.94 \\
\hline Mr & 698 & 51892 & 71808 & 0.7227 & 12.06 \\
\hline Mrs & 334 & 26118 & 34170 & 0.7644 & 13.65 \\
\hline father & 368 & 28912 & 37740 & 0.7661 & 13.71 \\
\hline
\end{tabular}

As in French, prepositions, articles, conjunctions, and auxiliary verbs produce the smallest distances between texts. In other words, they are common to all authors, or, similarly, the structure of the language imposes rigid restraints on those who use it; therefore authors can hardly be differentiated by means of these words. Individual differences are therefore, on average, very weak.

By contrast, personal pronouns, verbs, and the most ordinary nouns (substantive, adjectives) are more variable in their usage among different texts and authors. That is the case in French as well.

A main conclusion can be drawn from the analysis of distance contributions: for authorship attribution, a great number of experiments like this may lead to a set of more or less frequent words able to discriminate authors in general. Texts of different length will then become comparable. One can understandably be sceptical about this outcome, but one can imagine how to validate the idea, applying the procedure to a great number of texts which are of established authorship. Then one could apply these lists to unknown or doubtful texts. It would be a good supplement to a standardised scale for authorship attribution. 


\subsection{A provisional scale for authorship attribution}

The reasoning above postulates that the indices of inter-textual distance calculated between texts by a same author must be significantly lower than those between texts by different authors. The results of the blind-test appear to validate this postulate but nevertheless a more detailed examination is of interest. Given the choices made by G. Ledger, there are 153 different pairs of excerpts by the same authors and 1173 pairs which group excerpts by different authors. Let the entire 1,326 indices be ranked by ascending values. Appendix 2 reproduces the beginning and the ending tails of this distribution. Fig. 4 presents their repartition in equal classes (absolute densities).

Fig. 4 Distribution of inter-textual distance indices ranked by ascending values in equal classes from Oxquarry1.

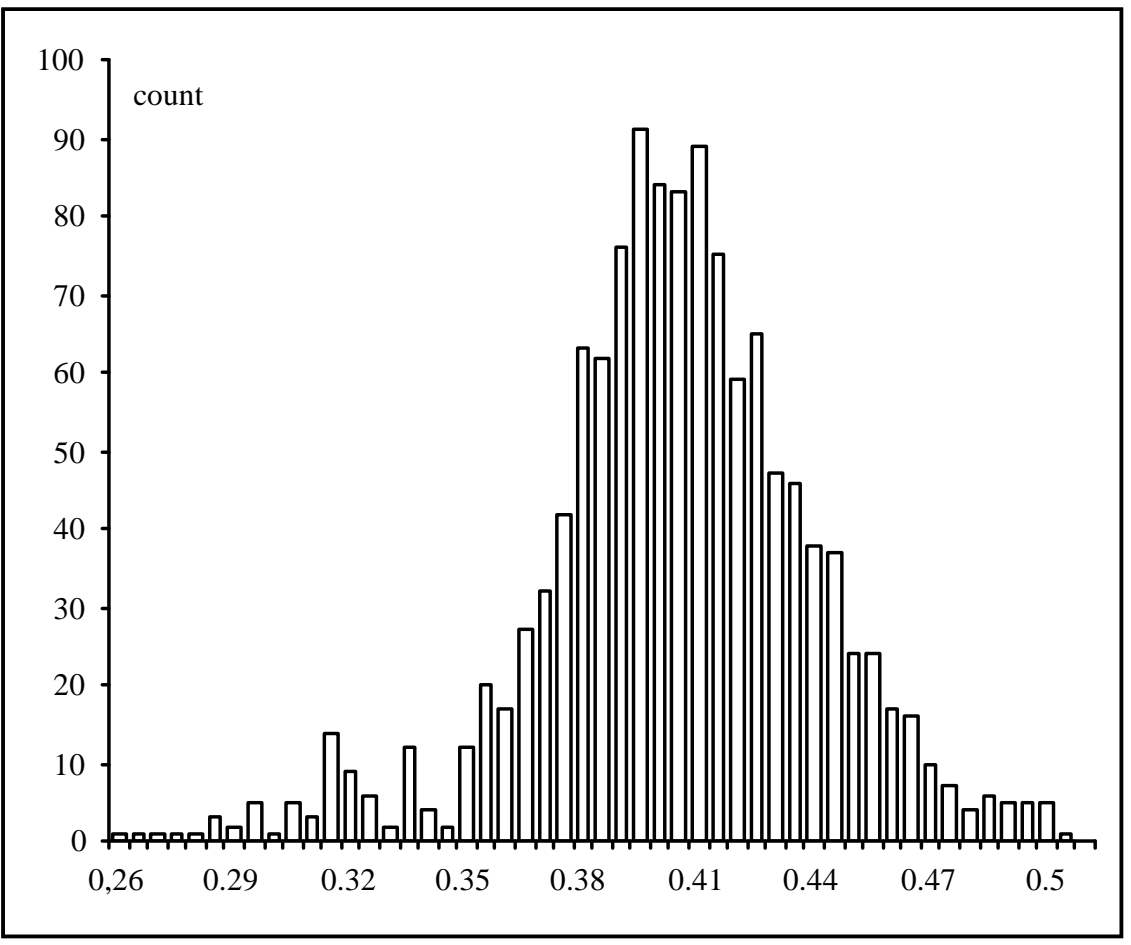

The shape of this graph suggests clearly a mix of different populations :

— in the middle of the graph, a large population, with a "mode" around 0.395-0.410 seems to be more or less "Gaussian"; 
— on the right tail, a little group of text pairs separated by distance indices over 0.48 . Appendix 2.4 highlights the singularity of Conrad and suggests that, for some these texts, a supplementary factor (genre?) adds its effects to differences in authorship and content.

— on the left tail, two small populations of which one cannot say if their distribution is Gaussian or not because they are of insufficient size. The first population from the left has a mode at $0.315-0.320$; the second population mode seems to take place at $0.325-0.330$. Appendix 2.1 indicates clearly the difference between these two populations. The first one is quite exclusively composed of text pairs drawn from the same books; the second one contains a majority of excerpts from two different books by the same author.

Of course these populations are not strictly separated, but some thresholds appear clearly: — all indices less than .349 are within excerpts by the same author. $56 \%$ of all distances between texts by the same author are below this threshold (Appendix 2.1).

— between .35 and .36 one can find a great majority of pairs of texts by the same author (Appendix 2.2, marked in bold) and very few of them can be found over .36 and under .395 (Appendix 2.3).

— all indices above .395 are between excerpts by different authors : $78 \%$ of all pairs of texts by two different authors are above this threshold.

It is also noticeable that the highest indices (Appendix 2.3) observed for the same author, concern for most part only different books, except Orczy's Scarlet Pimpernel (.364) and Elusive P. (.376), Conrad 's Lord Jim (.367 and 373) and Almayer's Foly (.384). For these four books, various explanations are possible, for example: they are more "heterogeneous" than the others; G. Ledger chose excerpts very different from each other.

A final remark: in Corpus Oxquarry1, the texts are of equal lengths. Are the results equally good when the method is applied to texts the lengths of which are different? In other words, what is the influence of text length on inter-textual distance? And, if there is an actual influence, how could one neutralise this drawback? 


\section{INFLUENCE OF TEXT LENGTHS ON DISTANCE INDEX}

Firstly, a modification of formula (1) is presented to adapt it in the case of texts unequal in length. To measure the potential bias due to the differences of length, several trials were carried out. These experiments followed the same principle: the length was the only factor allowed to vary while the other factors were neutralised (as strictly as possible).

\subsection{Calculation of Inter-textual distance index on texts of different sizes}

The extended reasoning is presented in Labbé \& Labbé (2001 and 2003). It is proposed to "reduce" the larger text $(B)$ to the size of the shorter one $(A)$ as explained below. Let $N_{a}$ and $N_{b}$ be the size of the two texts (in tokens), and a type $i$ of which absolute frequencies are $f_{i a}$ and $f_{i b}$ in $A$ and $B$. The mathematical expectancy of this type in $A$, considering its frequency in $B\left(f_{i b}\right)$ is:

$$
E_{i a(u)}=F_{i b} * u \text { with } u=\frac{N_{a}}{N_{b}}
$$

and the "absolute" distance is: $F_{i a}-E_{i a(u)}$

Thus, the new distance index between $A$ and $B$ is obtained in substituting $E_{i a(u)}$ for $F_{i b}$ in the formula (1):

$$
\text { (3) } \operatorname{Drel}_{(A, B)}=\frac{\sum_{\mathrm{i} \in(\mathrm{A}, \mathrm{B})}\left|\mathrm{F}_{\mathrm{ia}}-\mathrm{E}_{\mathrm{ia}(\mathrm{u})}\right|}{2 N_{a}}
$$

Two main experiments were carried out in order to test the accuracy of the formula (3) and to measure its possible sensitivity to the differences of text lengths. First, the Corpus Oxquarry1 was used, then a larger one, Oxquarry2, was collected with the help of G. Ledger. 


\subsection{Experiment on short texts (Oxquarry1)}

Given three texts $A, B, C$, when two of them $(A, B)$ are joined $(A \cup B)$, is the new distance index between this group and any other text $(\mathrm{C})$, equal to the arithmetic mean of the original distances (see formula (2) above)? In what follows, the indices calculated in applying formula (2) are designated "theoretical", and those using formula (3) on the actual "new text" created by joining $A$ to $B$ are designated "observed". If the results of the two calculations are equal, it mean that the calculation is insensitive to differences in text lengths.

In the blind-text presented above,

— the two nearest texts are excerpts from Stevenson's Catrionae (1D and 2W). These two excerpts were joined and this "new text" was designated "Stevenson2" (since the length of this "text" is 20,000 tokens, the double of the other excerpts which are unchanged);

— the most distant excerpts written by a single author are 1I (Orczy, Elusive) and 1U (Orczy, Scarlet). They were also joined ("Orczy2").

The theoretical and observed indices are calculated between these two "new" texts $(20,000$ tokens long) and the 48 remaining excerpts (10,000 tokens long). Table 6 below gives the results of the comparison between the two results.

This comparison reveals:

— a systematic bias: observed indices are always less than the theoretical ones. Almost all the discrepancies vary between $2 \%$ and $4 \%$, but they are above $5 \%$ in the case of two occurrences for Stevenson and in four for Orczy (bold characters in Table 6). Given the fact that for French texts of different lengths, the results fall within an uncertainty margin of 5\%, these six indices must be considered as "anomalous".

It is worth noting that, for Orczy, half of the discrepancies come from distances within her own work (between Elusive P. and Scarlet P.). More generally, the highest values in the last two columns are observed between texts by the same author or between the nearest texts by different authors. 
Table 6 Influence of text size on distance indices (Corpus Oxquarry1, scale difference 1:2)

\begin{tabular}{|c|c|c|c|c|c|c|}
\hline & \multicolumn{2}{|c|}{ Theoretical index } & \multicolumn{2}{|c|}{ Observed index } & \multicolumn{2}{|c|}{ Difference $\%$} \\
\hline & Stevenson2 & Orczy2 & Stevenson2 & Orczy2 & Stevenson2 & Orczy2 \\
\hline 1A (Hardy Jude) & 0.4384 & 0.4228 & 0.4267 & 0.4057 & -2.7 & -4.0 \\
\hline 1B (Butler Erewhon revisit.) & 0.4164 & 0.4394 & 0.4081 & 0.4265 & -2.0 & -2.9 \\
\hline 1C (Morris News) & 0.4122 & 0.4355 & 0.4034 & 0.4219 & -2.1 & -3.1 \\
\hline 1E (Butler Erewhon revisit.) & 0.3962 & 0.4424 & 0.3836 & 0.4299 & -3.2 & -2.8 \\
\hline 1F (Stevenson Ballantrae) & 0.3315 & 0.4058 & 0.3158 & 0.3857 & -4.7 & -5.0 \\
\hline 1G (Conrad Lord Jim) & 0.3879 & 0.4343 & 0.3776 & 0.4171 & -2.7 & -4.0 \\
\hline 1H (Hardy Madding) & 0.4138 & 0.4145 & 0.4037 & 0.3969 & -2.4 & -4.3 \\
\hline 1J (Morris Dream of JB) & 0.4256 & 0.4560 & 0.4143 & 0.4418 & -2.7 & -3.1 \\
\hline 1L (Hardy Jude) & 0.2842 & 0.4378 & 0.2644 & 0.4231 & -7.0 & -3.4 \\
\hline 1M (Orczy Scarlet P) & 0.4052 & 0.4197 & 0.3957 & 0.4027 & -2.4 & -4.1 \\
\hline 1N (Stevenson Ballantrae) & 0.4353 & 0.4034 & 0.4268 & 0.3841 & -2.0 & -4.8 \\
\hline 10 (Conrad Lord Jim) & 0.3256 & 0.4237 & 0.3106 & 0.4056 & -4.6 & -4.3 \\
\hline 1P (Chesterton Man who was) & 0.4290 & 0.4304 & 0.4194 & 0.4153 & -2.2 & -3.5 \\
\hline 1Q (Butler Erewhon revisit.) & 0.4373 & 0.4207 & 0.4288 & 0.4036 & -1.9 & -4.1 \\
\hline 1R (Chesterton Man who was) & 0.3915 & 0.4327 & 0.3810 & 0.4173 & -2.7 & -3.6 \\
\hline 1S (Morris News) & 0.4059 & 0.4155 & 0.3963 & 0.3980 & -2.4 & -4.2 \\
\hline 1T (Conrad Almayer) & 0.3572 & 0.4279 & 0.3459 & 0.4138 & -3.1 & -3.3 \\
\hline 1V (Conrad Lord Jim) & 0.5118 & 0.4612 & 0.5029 & 0.4456 & -1.7 & -3.4 \\
\hline 1W (Orczy Elusive P) & 0.4764 & 0.4365 & 0.4679 & 0.4187 & -1.8 & -4.1 \\
\hline 1X (Hardy Wessex Tales) & 0.4346 & 0.3925 & 0.4242 & 0.3705 & -2.4 & -5.6 \\
\hline 1Y (Tressel Ragged TP) & 0.4157 & 0.4173 & 0.4037 & 0.3991 & -2.9 & -4.4 \\
\hline 1Z (Tressel Ragged TP) & 0.4413 & 0.4249 & 0.4319 & 0.4050 & -2.1 & -4.7 \\
\hline 2A (Butler Erewhon revisit.) & 0.4325 & 0.4295 & 0.4215 & 0.4148 & -2.5 & -3.4 \\
\hline 2B (Morris Dream of JB) & 0.4177 & 0.4299 & 0.4086 & 0.4127 & -2.2 & -4.0 \\
\hline 2C (Tressel Ragged TP) & 0.4330 & 0.4290 & 0.4246 & 0.4151 & -1.9 & -3.2 \\
\hline 2D (Hardy Jude) & 0.4184 & 0.4263 & 0.4100 & 0.4115 & -2.0 & -3.5 \\
\hline 2E (Stevenson Ballantrae) & 0.4148 & 0.4064 & 0.4038 & 0.3871 & -2.7 & -4.7 \\
\hline 2F (Hardy Wessex Tales) & 0.3244 & 0.4148 & 0.3112 & 0.3958 & -4.1 & -4.6 \\
\hline 2G (Orczy Elusive P) & 0.4284 & 0.4306 & 0.4169 & 0.4138 & -2.7 & -3.9 \\
\hline 2H (Conrad Lord Jim) & 0.4599 & 0.3977 & 0.4501 & 0.3747 & -2.1 & -5.8 \\
\hline 2I (Morris News) & 0.4033 & 0.4181 & 0.3932 & 0.4006 & -2.5 & -4.2 \\
\hline 2J (Hardy Well beloved) & 0.3902 & 0.4334 & 0.3793 & 0.4192 & -2.8 & -3.3 \\
\hline 2K (Conrad Almayer) & 0.4057 & 0.4138 & 0.3948 & 0.3967 & -2.7 & -4.1 \\
\hline 2L (Hardy Well beloved) & 0.4861 & 0.4379 & 0.4773 & 0.4227 & -1.8 & -3.5 \\
\hline 2M (Morris News) & 0.3983 & 0.4237 & 0.3877 & 0.4065 & -2.6 & -4.1 \\
\hline 2N (Conrad Almayer) & 0.3810 & 0.4260 & 0.3686 & 0.4125 & -3.3 & -3.2 \\
\hline 20 (Forster Room with view) & 0.4546 & 0.4224 & 0.4474 & 0.4055 & -1.6 & -4.0 \\
\hline 2P (Forster Room with view) & 0.3977 & 0.4316 & 0.3878 & 0.4139 & -2.5 & -4.1 \\
\hline 2Q (Conrad Almayer) & 0.4245 & 0.4235 & 0.4135 & 0.4055 & -2.6 & -4.2 \\
\hline 2R (Stevenson Catrionae) & 0.4339 & 0.4207 & 0.4265 & 0.4037 & -1.7 & -4.0 \\
\hline 2S (Hardy Madding) & 0.2966 & 0.4154 & 0.2788 & 0.3949 & -6.0 & -4.9 \\
\hline 2T (Hardy Well beloved) & 0.4076 & 0.4163 & 0.3962 & 0.3990 & -2.8 & -4.2 \\
\hline 2U (Chesterton Man who was) & 0.4240 & 0.4182 & 0.4136 & 0.3989 & -2.4 & -4.6 \\
\hline 2V (Forster Room with view) & 0.4293 & 0.4249 & 0.4196 & 0.4071 & -2.2 & -4.2 \\
\hline 2W (Stevenson Catrionae) & 0.4098 & 0.4395 & 0.3992 & 0.4211 & -2.6 & -4.2 \\
\hline 2X (Hardy Well beloved) & 0.4336 & 0.4264 & 0.4240 & 0.4096 & -2.2 & -3.9 \\
\hline 2Y (Orczy Scarlet P) & 0.4386 & 0.3668 & 0.4286 & 0.3400 & -2.3 & -7.3 \\
\hline 2Z (Hardy Madding) & 0.4226 & 0.4154 & 0.4111 & 0.3969 & -2.7 & -4.5 \\
\hline Mean & & & & & -2.5 & -4.2 \\
\hline
\end{tabular}


The same experiment was repeated:

— step 3: the closest excerpt was added to Stevenson2 (i.e. $1 \mathrm{~K}$ which is also an excerpt from $\underline{\text { Catrionae }})$ in order to constitute "Stevenson3" and to Orczy2 (1M, $\underline{\text { Scarlett P. }}$ ) in order to constitute "Orczy3";

— step 4: 2R is added to Stevenson3 (Stevenson4) and 1W to Orczy3 (Orczy4);

— steps $5 \& 6$. Stevenson 5 and 6 are formed by merging with Stevenson $4: 1 F$ and then $1 \mathrm{~N}$; Orczy5 and 6 are constituted by merging with Orczy4: $2 \mathrm{G}$ and then $2 \mathrm{Y}$.

It is not possible to proceed further in this way because there are only six excerpts by Orczy. The distance indices between these groups and the remaining 10,000 token length excerpts were calculated. This experiment highlights index behaviour when calculated with texts the sizes of which diverge further and further. Tables 7 and 8 below show the results of this new experiment.

Table 7 Stevenson. Comparison with all remaining 10,000 token excerpts Means of the whole indices

\begin{tabular}{|l|c|c|c|}
\hline & $\begin{array}{c}\text { A } \\
\text { Means of Observed } \\
\text { Indices }\end{array}$ & $\begin{array}{c}\text { B } \\
\text { Means of Theoretical } \\
\text { Indices }\end{array}$ & (A-B)/B\% \\
\hline Stevenson 20,000 & .402 & .412 & -2.5 \\
\hline Stevenson 30,000 & .397 & .413 & -3.9 \\
\hline Stevenson 40,000 & .379 & .412 & -8.0 \\
\hline Stevenson 50,000 & .374 & .406 & -7.9 \\
\hline Stevenson 60,000 & .372 & .405 & -8.2 \\
\hline
\end{tabular}

Table 8 Orczy. Comparison with all remaining 10,000 token excerpts Means of the whole indices

\begin{tabular}{|l|c|c|c|}
\hline & $\begin{array}{c}\text { A } \\
\text { Means of Observed } \\
\text { Indices }\end{array}$ & $\begin{array}{c}\text { B } \\
\text { Means of Theoretical } \\
\text { Indices }\end{array}$ & (A-B)/B\% \\
\hline Orczy 20,000 & .406 & .424 & -4.2 \\
\hline Orczy 30,000 & .390 & .421 & -7.3 \\
\hline Orczy 40,000 & .387 & .420 & -7.9 \\
\hline Orczy 50,000 & .392 & .425 & -7.9 \\
\hline Orczy 60,000 & .391 & .426 & -8.2 \\
\hline
\end{tabular}

The systematic bias noted above appears clearly. It is also clear that the relation between text lengths and distance indices is non-linear. As the differences of size between texts increase, the growth rate of the divergences between theoretical and observed values decreases (last column). The same phenomenon was noted with the French corpora but to a 
lesser degree (Labbé \& Labbé, 2003). Again, these differences stem from a lesser proportion of low frequency word types in French corpora (due to lemmatisation).

This bias entails an obvious consequence: when comparing texts the sizes of which greatly differ, the indices calculated in applying Formula 3 to the longest ones will tend to be lower than those calculated with the shortest ones.

However, two objections may be made to this experiment: the excerpts by Stevenson are much nearer one another than those by Orczy, and it can be added that the larger the "artificial" texts, the lesser the others : they remain 48 when length factor is equal to 2; 40 when it reaches 6 (it is the reason why values in column B vary).

In order to organise a more systematic experiment, G. Ledger was asked to set up a new corpus.

\subsection{First experiment on long texts (Corpus Oxquarry2)}

An agreement with Dr Ledger was reached about the main characteristics of this new corpus. It should contain the entire novels from which the Corpus Oxquarry1 was composed in order to make the results comparable. And it was decided to add other novels written during the same period (1870-1914) by other writers. The main purpose was to obtain, by a choice independent of the operator, the largest possible range of sizes for texts written in the same genre, period and by undisputed authors.

The result of this selection is Corpus Oxquarry2 (Appendix 3). This corpus is 5.1 million tokens long (ten times Oxquarry1) with an average length slightly over 100,000 tokens. The length ratio is nearly 1:16 between Heart of Darkness by Joseph Conrad (20,243 tokens) and Middlemarch by George Eliot (316,156 tokens). This ratio of lengths clearly exceeds the limits of validity of formula (3) observed by Labbé and Labbé (2003). Beyond a scale of 1:10, the experiments conducted on French corpora demonstrate that distance indices are biased, especially when calculations include the longest and the shortest texts.

This bias should not be over-emphasized. For example, tree-classification - applied to the whole matrix of indices calculated on Corpus Oxquarry2 applying Formula 3 - is without error (Fig. 5 below). Of course, the lengths of the paths must be carefully considered. For example, for Conrad's cluster, some novels appear to be very near one another (Lord Jim, Arrow of Gold or Victory), while others are more outlying (Almayer's Folly, Typhoon, Heart of Darkness). One must not draw conclusions too quickly about Conrad's work because the first set contains much longer texts than the second one. In fact, the first experiment above 
allows one to "predict" that, the influence of the other factors being equal, the longer texts will be relatively nearer all the others than the shorter ones.

Fig. 5. Tree classification of Corpus Oxquarry2 applying Formula 3

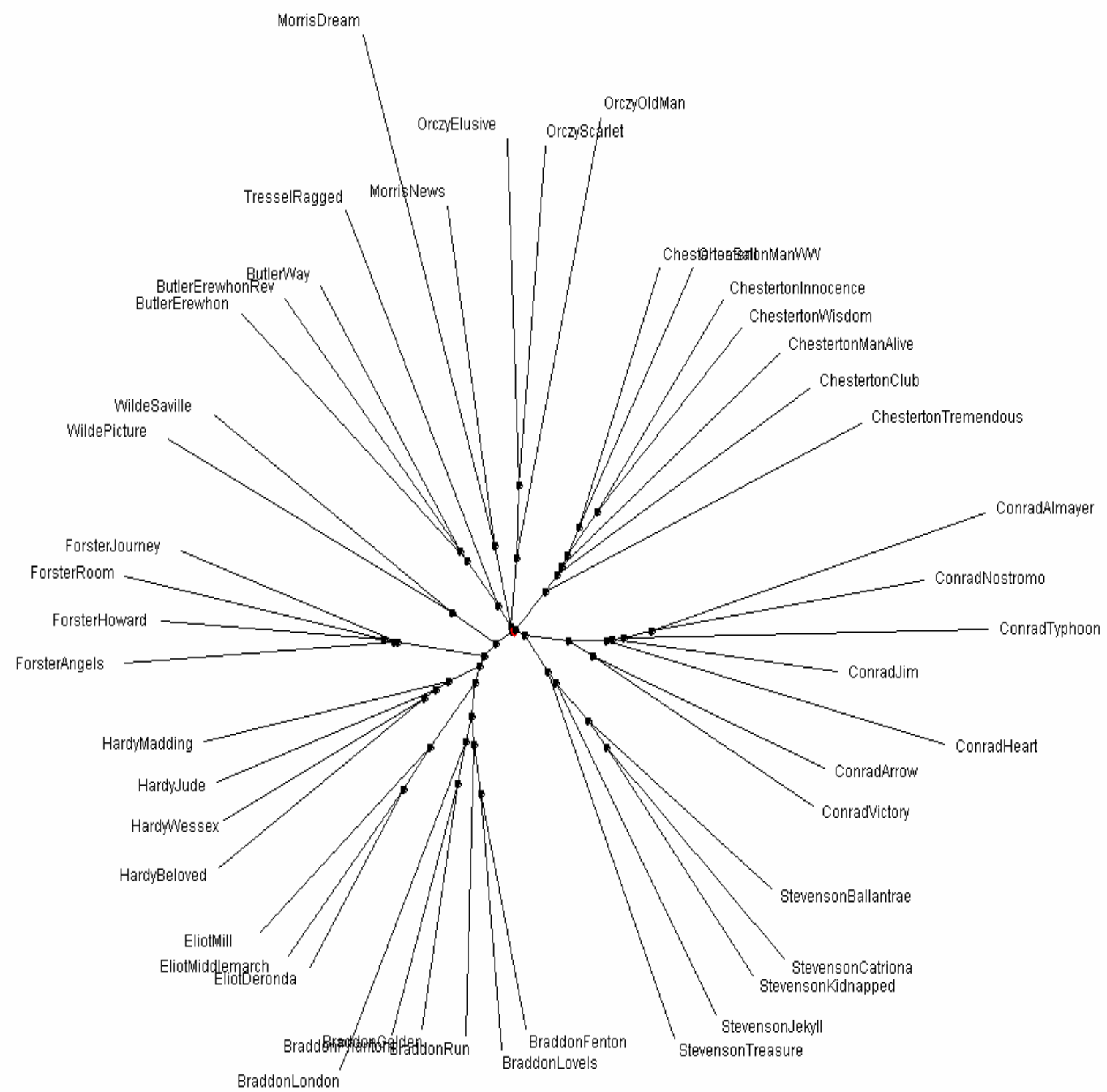

Even if tree-classification appears to be accurate, one must bear in mind that this classification does not yield "proof" of authorship, but only provides the "best fit" of the whole population. Its suggestions must be confirmed by the direct examination of the matrix as in the blind-test presented above. Here, the mean distance index is equal to .315 , greatly below the one obtained for Oxquarry1 (.405). In other words, when the mean sizes of the texts are multiplied by ten, their distance indices decrease by nearly $20 \%$ on average.

But this decrease is not linear and strictly proportional. There are also some irregularities which render the method presented above no longer reliable for authorship attribution. The 
$5 \%$ lower limit (mean less 1.96 standard deviations) is equal to .237; the upper limit to 394 .

All pairs of novels separated by a distance greater than .394 are indeed by two different authors. By contrast, the lower boundary generates three errors of attribution (bold type in Table 9).

Table 9. Inter-textual distance indices calculated on Corpus Oxquarry2 applying formula 3 Pairs of distance indices which suggest same authorship (mean less 1.96 standard deviations)

\begin{tabular}{|c|c|c|}
\hline Text 1 & Text 2 & Indice \\
\hline EliotDeronda & EliotMiddlemarch & 0.155 \\
\hline BraddonFenton & BraddonLovels & 0.180 \\
\hline BraddonGolden & BraddonPhantom & 0.185 \\
\hline ChestertonInnocence & ChestertonWisdom & 0.186 \\
\hline EliotMiddlemarch & EliotMill & 0.196 \\
\hline EliotDeronda & EliotMill & 0.196 \\
\hline ForsterHoward & ForsterJourney & 0.202 \\
\hline BraddonFenton & BraddonRun & 0.204 \\
\hline ConradJim & ConradVictory & 0.204 \\
\hline BraddonGolden & BraddonLovels & 0.205 \\
\hline HardyJude & HardyWessex & 0.206 \\
\hline ChestertonBall & ChestertonManWW & 0.206 \\
\hline HardyBeloved & HardyWessex & 0.207 \\
\hline HardyMadding & HardyWessex & 0.208 \\
\hline BraddonLovels & BraddonPhantom & 0.211 \\
\hline ForsterHoward & ForsterRoom & 0.214 \\
\hline StevensonCatriona & StevensonKidnapped & 0.214 \\
\hline HardyBeloved & HardyJude & 0.216 \\
\hline StevensonBallantrae & StevensonKidnapped & 0.219 \\
\hline HardyJude & HardyMadding & 0.219 \\
\hline ChestertonBall & ChestertonInnocence & 0.219 \\
\hline ForsterAngels & ForsterJourney & 0.219 \\
\hline ConradArrow & ConradVictory & 0.220 \\
\hline StevensonBallantrae & StevensonCatriona & 0.221 \\
\hline BraddonFenton & BraddonGolden & 0.222 \\
\hline BraddonLovels & BraddonRun & 0.222 \\
\hline ForsterJourney & ForsterRoom & 0.224 \\
\hline ChestertonInnocence & ChestertonManWW & 0.226 \\
\hline ForsterAngels & ForsterHoward & 0.228 \\
\hline ChestertonBall & ChestertonWisdom & 0.229 \\
\hline BraddonLondon & BraddonPhantom & 0.230 \\
\hline BraddonLovels & EliotDeronda & 0.230 \\
\hline ForsterAngels & ForsterRoom & 0.231 \\
\hline BraddonFenton & EliotMiddlemarch & 0.231 \\
\hline ButlerErewhonRev & ButlerWay & 0.232 \\
\hline ChestertonBall & ChestertonManAlive & 0.233 \\
\hline EliotMill & HardyJude & 0.234 \\
\hline BraddonFenton & BraddonPhantom & 0.234 \\
\hline BraddonLovels & EliotMiddlemarch & 0.235 \\
\hline BraddonGolden & BraddonRun & 0.236 \\
\hline BraddonPhantom & BraddonRun & 0.237 \\
\hline ChestertonManWW & ChestertonWisdom & 0.237 \\
\hline
\end{tabular}


As predicted from the discussion above, these errors involve the longest texts. Indeed, it is possible that the four novels and the two authors (Braddon and Eliot) were in fact "neighbours" as to their content and vocabularies, but also it is clear that Formula (3) - when applied to texts the size of which differs greatly (here more than 1:15) — "advantages" the longer ones, ie: the longer ones appear to be nearer one another - and also from all the others — than the shorter ones.

In order to measure the strength of association between distance index and length of the texts examined, a calculation is proposed. The comparison of each text with the others generates 47 indices. If the Formula 3 "advantages" the longer texts, the averages of their 47 indices must be lower than the same averages calculated for the shorter ones. The results of all these calculations are summarised in Fig. 6.

Fig. 6. Correlation between the length of each text and its average distance index compared with all the other texts (Corpus Oxquarry2).

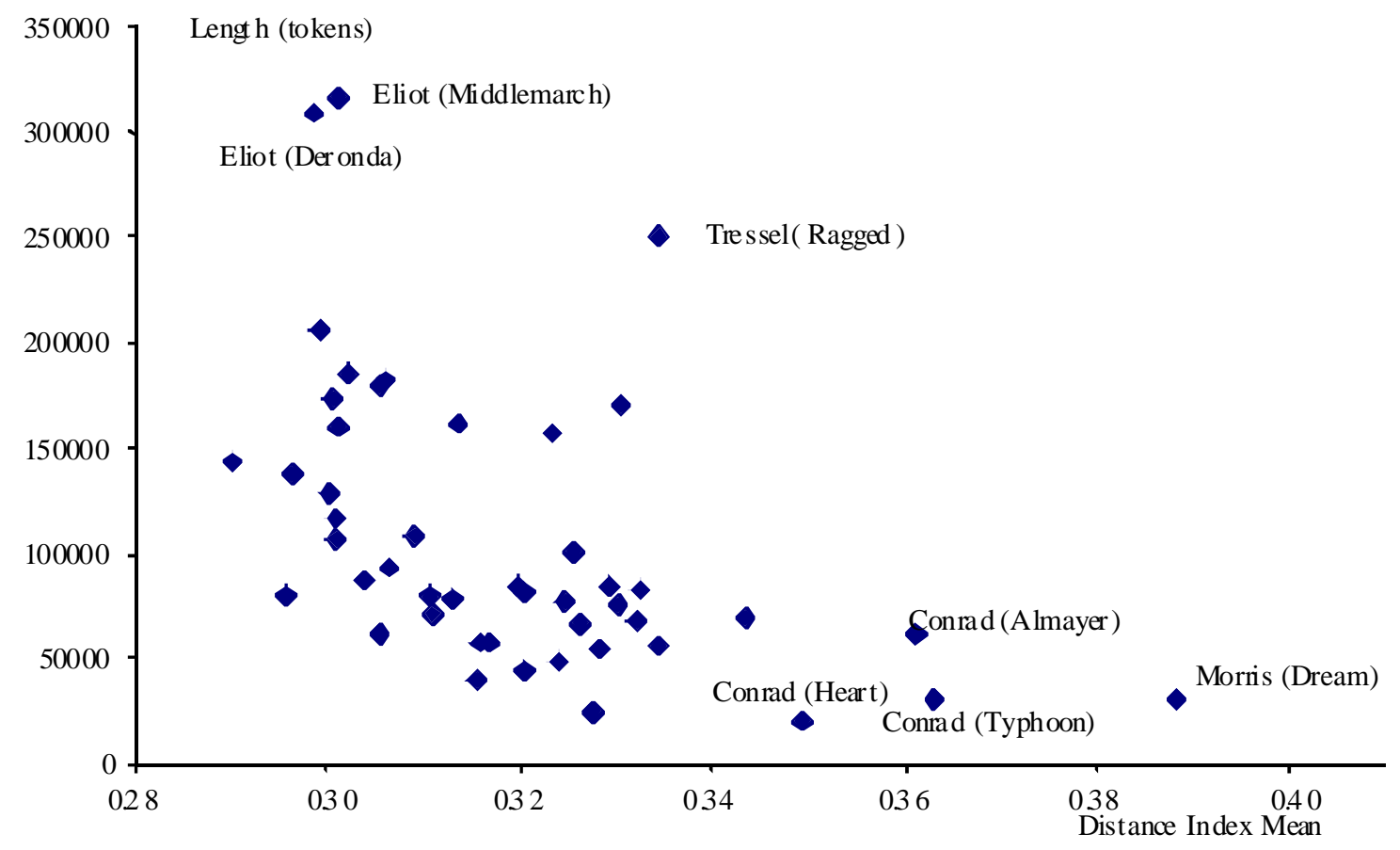

Fig. 6 suggests an inverse relation between lengths of texts and distance indices. The plotted points are oriented NW-SE and present a more or less hyperbolic shape: the shorter the texts, the farther apart they appear to be in relation with the others. But it is noticeable that 7 texts stand out apart from the others. On the lower right, four of the shortest texts: Dream of $\underline{\text { J.B }}$ by Morris and three novels by Conrad (Typhoon, Almayer's Folly, Heart of Darkness); on the upper left, the three longer texts: Tressel's Ragged Philantropists, Eliot's $\underline{\text { Middlemarch and }}$ 
Daniel Deronda. When these 7 texts are removed, there is no longer a significant negative correlation between length of each text and the averaged distance indices. Of course the "size factor" is already present in these data, but it is "masked" by stronger factors: authorship and content. It is note-worthy that, once these 7 texts are extracted, the ratio between the shortest remaining text (Stevenson's Doctor Jekyll) and the longest remaining one (Braddon's The Mill) is equal to $1: 8$. Thus it is possible to draw a provisional conclusion: the procedure of authorship attribution, presented above in the blind-test, should be used only when the lengths of the texts compared are within a ratio less than $1: 8$.

\subsection{Second large scale experiment}

In order to measure accurately the effect of text length on the distance index, an experiment was conducted on Corpus Oxquarry2. A large number (100) of slices of equal size (n contiguous tokens) was drawn at random from each text of Corpus Oxquarry2 and formula (1) was applied to all the different pairs formed with all the equivalent slices drawn from all the other texts. Thus, the distance index between a pair of texts was the mean of 10,000 different calculations (for the reasons for this choice, see below, section 2.6). The experiment started with an excerpt size of 5,000 tokens; then this length was increased by 1,000 tokens and so on... until 20,000 tokens (summary is given in Table 10). It was not possible to go further than 20,000 tokens because of the length of the shortest novel (Conrad's Heart of Darkness: 20,243 tokens). During the experiment, the minimum index was between Chesterton's The Innocence of Father Brown and The Wisdom of Father Brown (identity of authorship and proximity of the two topics). The maximum index was between Butler's Erewhon and Conrad's Almayer's Folly which is also not surprising because of the wide disparity of topics which adds its effect to the expected effect of differing authorship. 
Table 10 Main results of a large scale experiment on Corpus Oxquarry2

\begin{tabular}{|c|c|c|c|c|c|}
\hline $\begin{array}{c}\text { Excerpt sizes } \\
\text { (tokens) }\end{array}$ & $\begin{array}{c}\text { Mean } \\
\text { Distance Index }\end{array}$ & $\begin{array}{c}\text { Minimum } \\
\text { Distance Index }\end{array}$ & $\begin{array}{c}\text { Maximum } \\
\text { Distance Index }\end{array}$ & $\begin{array}{c}\text { Standard } \\
\text { deviation }\end{array}$ & $\begin{array}{c}\text { Variation } \\
\text { coefficient }\end{array}$ \\
\hline 5000 & 0.4578 & 0.3775 & 0.5474 & 0.0244 & 0.0532 \\
\hline 6000 & 0.4463 & 0.3657 & 0.5428 & 0.0256 & 0.0574 \\
\hline 7000 & 0.4365 & 0.3547 & 0.5361 & 0.0269 & 0.0617 \\
\hline 8000 & 0.4277 & 0.3459 & 0.5305 & 0.0271 & 0.0634 \\
\hline 9000 & 0.4207 & 0.3372 & 0.5265 & 0.0279 & 0.0664 \\
\hline 10000 & 0.4137 & 0.3300 & 0.5203 & 0.0279 & 0.0674 \\
\hline 11000 & 0.4090 & 0.3240 & 0.5200 & 0.0290 & 0.0710 \\
\hline 12000 & 0.4038 & 0.3166 & 0.5161 & 0.0293 & 0.0726 \\
\hline 13000 & 0.3996 & 0.3097 & 0.5135 & 0.0300 & 0.0750 \\
\hline 14000 & 0.3954 & 0.3046 & 0.5106 & 0.0303 & 0.0766 \\
\hline 15000 & 0.3916 & 0.2991 & 0.5075 & 0.0304 & 0.0777 \\
\hline 16000 & 0.3882 & 0.2941 & 0.5051 & 0.0309 & 0.0797 \\
\hline 17000 & 0.3850 & 0.2889 & 0.5031 & 0.0311 & 0.0809 \\
\hline 18000 & 0.3821 & 0.2845 & 0.5016 & 0.0316 & 0.0828 \\
\hline 19000 & 0.3794 & 0.2807 & 0.5002 & 0.0318 & 0.0838 \\
\hline 20000 & 0.3766 & 0.2768 & 0.4978 & 0.0320 & 0.0849 \\
\hline
\end{tabular}

The index mean slowly decreases as the length of the excerpts increases. As shown on Fig. 7 below, this movement is not linear but more or less hyperbolic. The slight irregularities in the curves are usual in any random process, even if the number of excerpts is large. One must also note that the origin of $\mathrm{Y}$-axis is not placed at zero but at .25 in order to highlight the fluctuations of the indices: the distance indices decrease slowly and the rate of decrease is becomes less as the size of excerpts increases. Conclusions of the first experiment, on excerpts by Stevenson and Orczy, are verified. It is also clear that the influence of the "size factor" is more acutely felt on short texts - especially those under 10,000 tokens - than on longer ones.

Another characteristic is highlighted by the graph: the range between minimum and maximum indices widens as excerpt size expands. This change does not derive from a small number of marginal texts; it applies to all the series as indicated by the evolution of the standard deviation. If the variation of excerpt size did not influence the dispersion of the distance indices around the mean, the "coefficient of variation" (ratio of standard deviation/mean) would be stable. On the contrary, this coefficient increases regularly (Fig. 8). In other words, when the scale of observation is reduced, differences between texts are slightly but significantly eroded. 
Fig. 7 Evolution of the Index Mean and the Min/Max Range in relation to size of excerpts

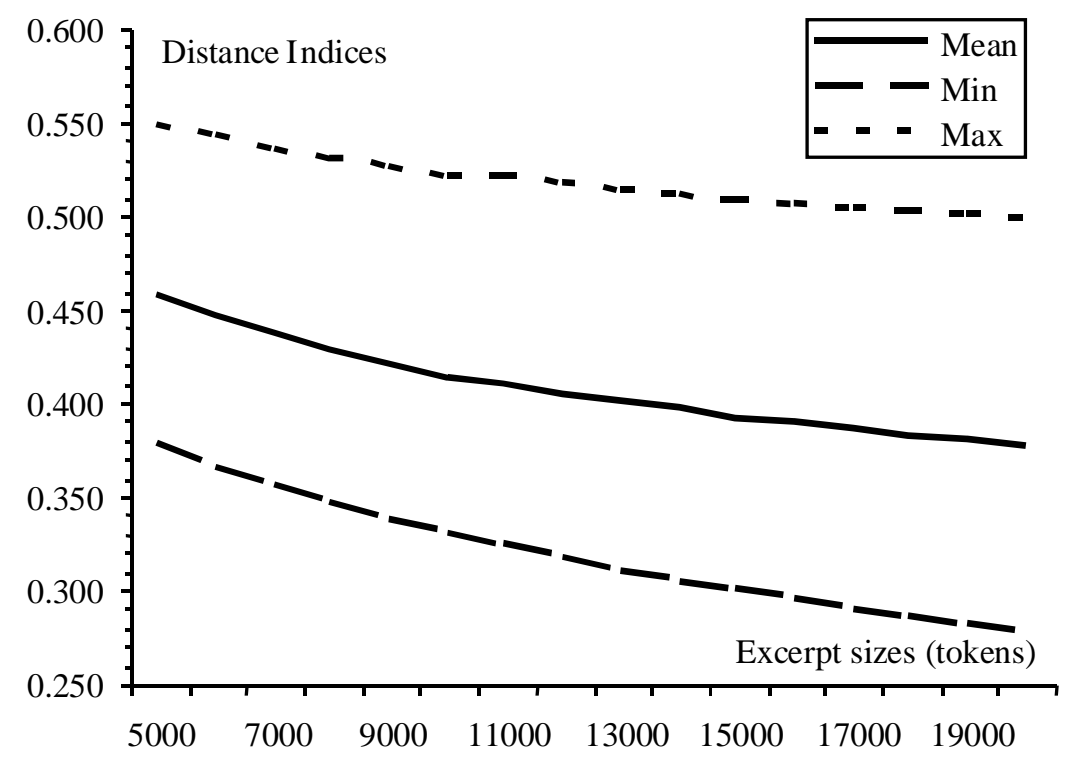

Fig. 8 Evolution of the ratio, Standard Deviation/Mean, related to the size of excerpts

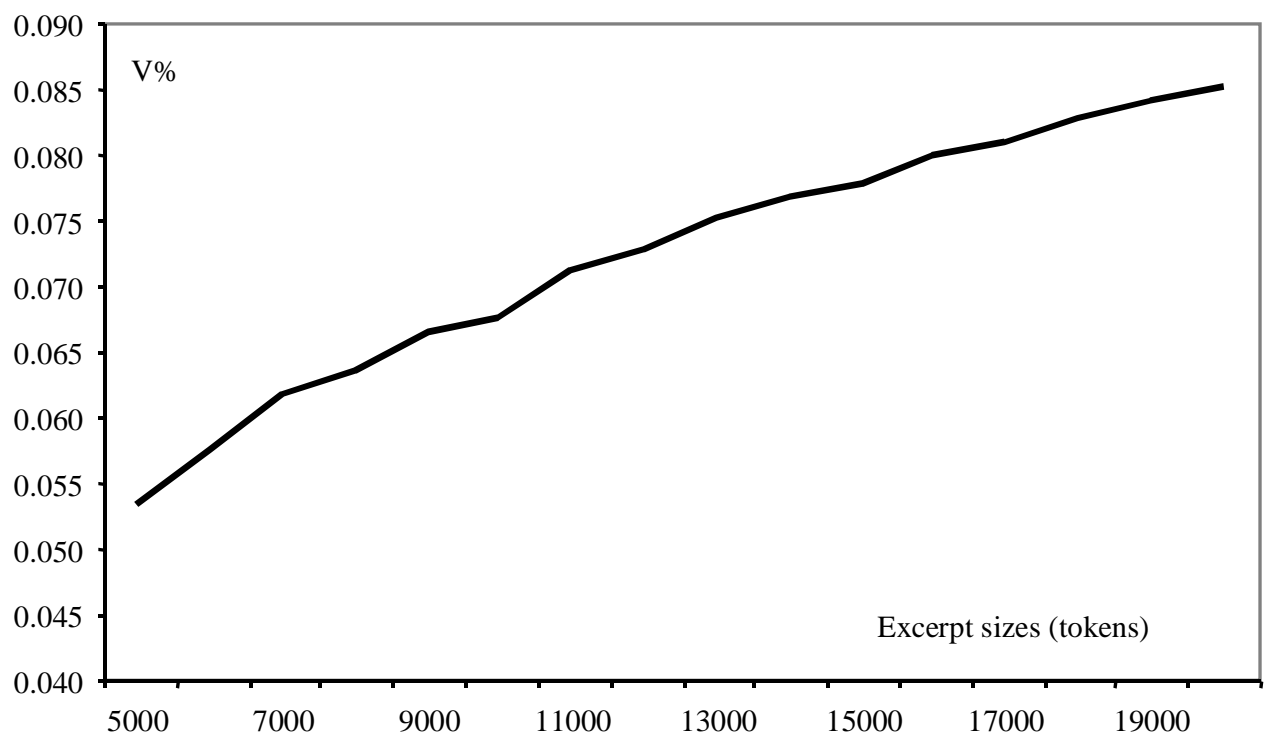

The main explanation is as follows: as the size of a text increases, the relative weight of the low frequency words decreases with respect to its whole vocabulary. Since, as shown above, the low frequency words contribute most to the distance index, the values of this index logically decrease as the text lengths increase. For French texts, lemmatisation greatly reduces the weight of these low frequency words. As a result, the influence of the length on the distance index is distinctly lower than with English texts. 


\subsection{Neutralisation of the length factor: the "sliding window method"}

How to neutralise the influence of the length factor in order to conduct authorship attribution experiments free from bias?

Formula (3) can be described as a process of drawing $\mathrm{N}_{\mathrm{a}}$ word types throughout the whole text B, B been greater than A (Fig. 9 below).

Fig. 9 How Formula 3 operates

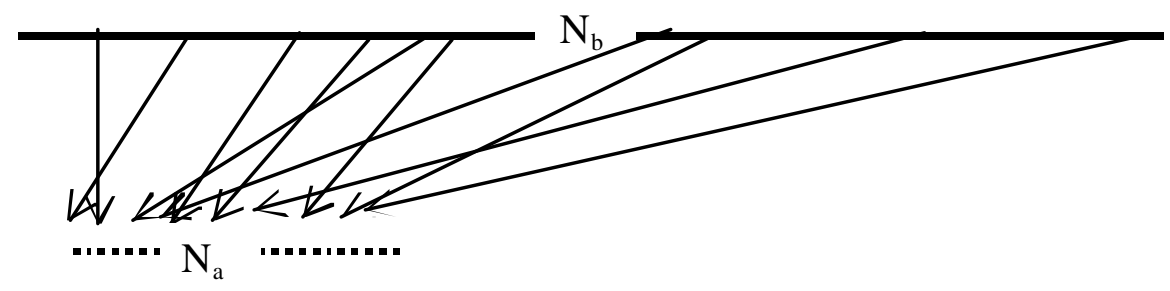

Applied to texts which were written in the same genre and period, this method usually "advantages" the longer text $(B)$ for reasons shown above. To obviate this drawback, Tom Merriam (2002b et 2003a) proposed to consider only one block of the $N_{a}$ first contiguous words drawn out of text $B$ (Fig. 10).

Fig. 10 Tom Merriam's method

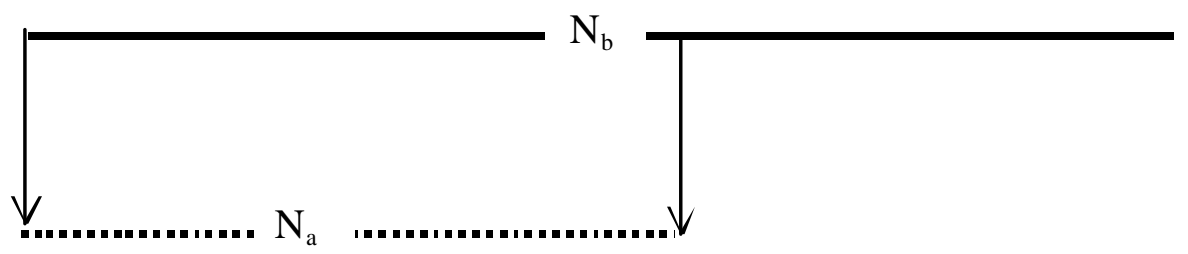

In T. Merriam's experiment, $\mathrm{N}_{\mathrm{a}}$ was equal or superior to the half of the lengths of the whole texts considered. Admittedly, he acknowledges that : "Problems might arise in the case of a [text] with a first half written by one author and a second by another" (Merriam, 2003a). These problems appear to have a simple solution: draw by chance out of $B$ a large number of excerpts of $N_{a}$ contiguous words and, then, calculate the means of all the distance indices between all these excerpts and the text $A$. This solutions entails several drawbacks. First, an error margin should be associated with the indices and this may often confound any clear conclusion. Above all, some interesting properties of Euclidian distances are endangered, like identity, symmetry or triangle inequality. 
In order to avoid these drawbacks, it is necessary that the calculation gives equal weight to each token in the two texts compared. With this aim in mind, it is proposed to use a "sliding window". For example, in the Fig. 11 below, the steps (or intervals) of the sliding windows are equal to $0.33 \mathrm{Na}$ : each token of $B$ is included three times in the calculation of the index.

Fig. 11 The "sliding window" method

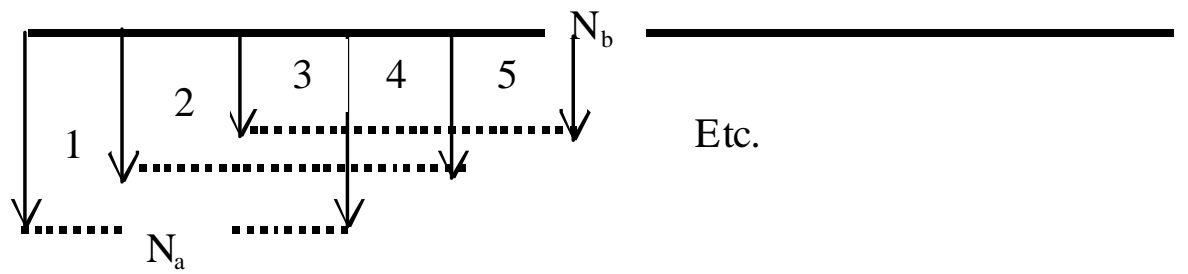

Actually, it is possible to chose a standardised length $(L)$ for these windows (or "blocks") of contiguous tokens. In which case, the distance index between two texts is the mean of all indices calculated with Formula (1) on all the excepts (of equal size) drawn from each of the two texts.

Are the results reliable when this method is applied to texts whose sizes greatly differ? Let:

$-A$ and $B$ be two texts the lengths of which are $N_{a}$ and $N_{b}$ tokens.

- $A$ and $B$ be divided into $M_{\mathrm{a}}$ and $\mathrm{M}_{\mathrm{b}}$ blocks of equal lengths (L contiguous tokens).

— their division be done along the two texts following the same interval of P tokens (see Figure 11 above), $L$ being a multiple of $\mathrm{P}$.

Formula (1) can be applied without bias to any pair of blocks, one of them $(i)$ drawn from $A$ and the other block $(j)$ drawn from $B$ - the length of these two blocks being equal to $L$ contiguous tokens - all the properties of an Euclidian distance are preserved, especially the ones of identity, symmetry and triangle inequality. The inter-textual distance index between $A$ and $B$ becomes the mean of all the different indices of distance which can be calculated between all differing blocks drawn from these two texts, ie $M_{a} \cdot M_{b}$ :

(4) $D_{L(a, b)}=\frac{1}{M_{a} \cdot M_{b}} \sum_{i=1}^{i=M a j=M_{b}} \sum_{j=1} D_{(a i, b j)}$ with $D_{(a i, b j)}$ calculated with (1)

In order to measure its accuracy, this procedure and formula were applied in a large number of experiments carried out on Corpus Oxquarry1 and 2. 


\subsection{Accuracy of the "sliding window" method.}

Corpus Oxquarry1 (made of texts the length of which is 10,000 tokens) was used in order to compare the results of the "sliding window" to those of the blind-test. Table 11 below presents a synthesis of the results of the experiments on blocks of 5,000 contiguous tokens, while varying the step interval.

\section{Table 11}

Synthesis of the results of experiment on Oxquarry 1, blocks of 5,000 contiguous tokens.

\begin{tabular}{|l|r|r|r|}
\hline Step interval in tokens & 1,000 & \multicolumn{1}{c|}{500} & \multicolumn{1}{c|}{200} \\
\hline Number of calculations (Ma.Mb) & 100 & 400 & 2,500 \\
Deviations between observed and expected values: & & & \\
$\quad$ Absolute mean & $0.62 \%$ & $0.59 \%$ & $0.61 \%$ \\
$\quad$ Standard deviation & $0.52 \%$ & $0.49 \%$ & $0.49 \%$ \\
Margin of error & $\pm 1.66 \%$ & $\pm 1.56 \%$ & $\pm 1.59 \%$ \\
\hline
\end{tabular}

When a step interval of 200 tokens is chosen (as in the last column of Table 11), it means that the first window starts at the first token and ends at the 5,000th token, the second starts at 201 and finishes at 5,200 and so on... until the last one which starts at 9,801 and finishes at 4,800 (the algorithm "buckles" in order to form the last blocks). Thus, each word of $A$ and $B$ appears in 50 different blocks of 5,000 tokens, and the distance index between each pair of texts $(A, B)$ is the mean of 2,500 calculations (each of the 50 blocks of $A$ is compared to each of the 50 blocks of $B$ ).

The results in Table 11 show that, in superimposing the two 1,326 cell matrices, the average deviation between expected and observed indices is appreciably less than $1 \%$ and that more than 19 twentieths of these discrepancies are contained within a fluctuation margin of $\pm 1.6 \%$ about this mean. In fact, 38 values are above or below these two thresholds. As predicted above, these anomalies derive from the nearest texts. For example, the greatest deviation $(-3.1 \%)$ was observed between $1 \mathrm{H}$ and $2 \mathrm{Z}$ (both excerpts from Far the madding crowd by Hardy); the next in order of magnitude $(+2.8 \%$ and $+2.6 \%)$ are from 4 excerpts from $\underline{\text { Catrionae }}$ by Stevenson. Two explanations can be offered. Firstly the experiments, presented in the first section of this paper, show that, in case of a great proximity in the vocabulary of the two texts, the augmentation of $L$ makes their intertextual distance decrease faster than the average, and conversely, when the two texts are farther apart (see also Fig. 6 
above). Stevenson's texts illustrate one case and Orczy's novels the other. Secondly, the buckle method adds a slight but real unaccounted - for variability.

Table 11, first line, shows the number of calculations entailed by the size of the step interval: this number grows exponentially as the step size is reduced. So a best compromise should be decided on, taking into consideration the demands of accuracy and the necessity of not waiting hours for results. Table 11 suggests that the step should be chosen in order to obtain at least 100 different calculations, and that no further accuracy can be expected in using over 400 different calculations.

Are these conclusions confirmed when applying the method to longer texts? Corpus Oxquarry2 was used in order to provide some answers.

Granted the fact that, in this corpus, the shortest text is slightly over 20,000 tokens, it was decided to adopt this size for $L$ and a $P$ (the size of the step) of 2,000 in order to ensure that the number of calculations between two texts are always greater than 100 . The average distance index is .3712 and the standard deviation .0282. So it can be supposed that all the novels which are separated by indices less than .315 (the mean less 1.96 standard deviations) are by the same authors. Table 12 below shows that this authorship attribution is free of error — contrary to Table 9 - but also that there is no text by Morris or by Wilde. One must bear in mind that, at this stage, the aim was not to give an answer for all the texts, but to avoid error. 
Table 12. Corpus Oxquarry2. Pairs of texts separated by an index lower than .315

(blocks of 20,000 tokens, step of 2,000 tokens).

\begin{tabular}{|l|l|c|}
\hline Text 1 & Text 2 & Indices \\
\hline ChestertonInnocence & ChestertonWisdom & 0.277 \\
HardyBeloved & HardyWessex & 0.282 \\
ForsterAngels & ForsterJourney & 0.283 \\
StevensonCatriona & StevensonKidnapped & 0.286 \\
ChestertonBall & ChestertonManWW & 0.287 \\
ForsterAngels & ForsterRoom & 0.289 \\
StevensonBallantrae & StevensonJekyll & 0.289 \\
StevensonBallantrae & StevensonKidnapped & 0.290 \\
ForsterHoward & ForsterJourney & 0.292 \\
ConradHeart & ConradJim & 0.292 \\
BraddonFenton & BraddonLovels & 0.292 \\
ForsterAngels & ForsterHoward & 0.292 \\
ForsterHoward & ForsterRoom & 0.292 \\
EliotDeronda & EliotMiddlemarch & 0.295 \\
StevensonBallantrae & StevensonCatriona & 0.295 \\
HardyBeloved & HardyJude & 0.297 \\
ChestertonBall & ChestertonInnocence & 0.299 \\
ForsterJourney & ForsterRoom & 0.299 \\
ChestertonInnocence & ChestertonManWW & 0.299 \\
StevensonKidnapped & StevensonTreasure & 0.300 \\
HardyJude & HardyWessex & 0.301 \\
HardyMadding & HardyWessex & 0.302 \\
ChestertonClub & ChestertonManWW & 0.303 \\
StevensonJekyll & StevensonKidnapped & 0.303 \\
ChestertonBall & ChestertonWisdom & 0.304 \\
ChestertonBall & ChestertonClub & 0.305 \\
ChestertonManWw & ChestertonWisdom & 0.306 \\
ConradJim & ConradVictory & 0.306 \\
ChestertonBall & ChestertonManAlive & 0.307 \\
ButlerErewhonRev & 0.308 \\
BraddonFenton & 0.310 \\
ConradArrow & ButlerWay & 0.310 \\
BraddonGolden & BraddonRun & 0.310 \\
ChestertonClub & ConradVictory & 0.312 \\
ChestertonManAlive & BraddonPhantom & 0.312 \\
ConradJim & ChestertonWisdom & 0.313 \\
ChestertonClub & ChestertonWisdom & 0.313 \\
ChestertonClub & 0.314 \\
BraddonGolden & ConradTyphoon & 0.315 \\
& ChestertonInnocence & \\
\hline & ChestertonManAlive & BraddonLovels \\
\hline
\end{tabular}

As was remarked about the blind-test with Oxquarry1, all the reasoning is based on two assumptions: (1) the corpus contains several authors and (2) there are, at least, two novels by each of these authors (this second assumption is not entirely true: there is only one book by Tressel).

Does tree-classification give a more complete and reliable answer? (Fig. 12) 
Fig. 12 Tree-classification of Corpus Oxquarry2 using Formula (4) (window size: 20,000 tokens; step: 2,000 tokens).

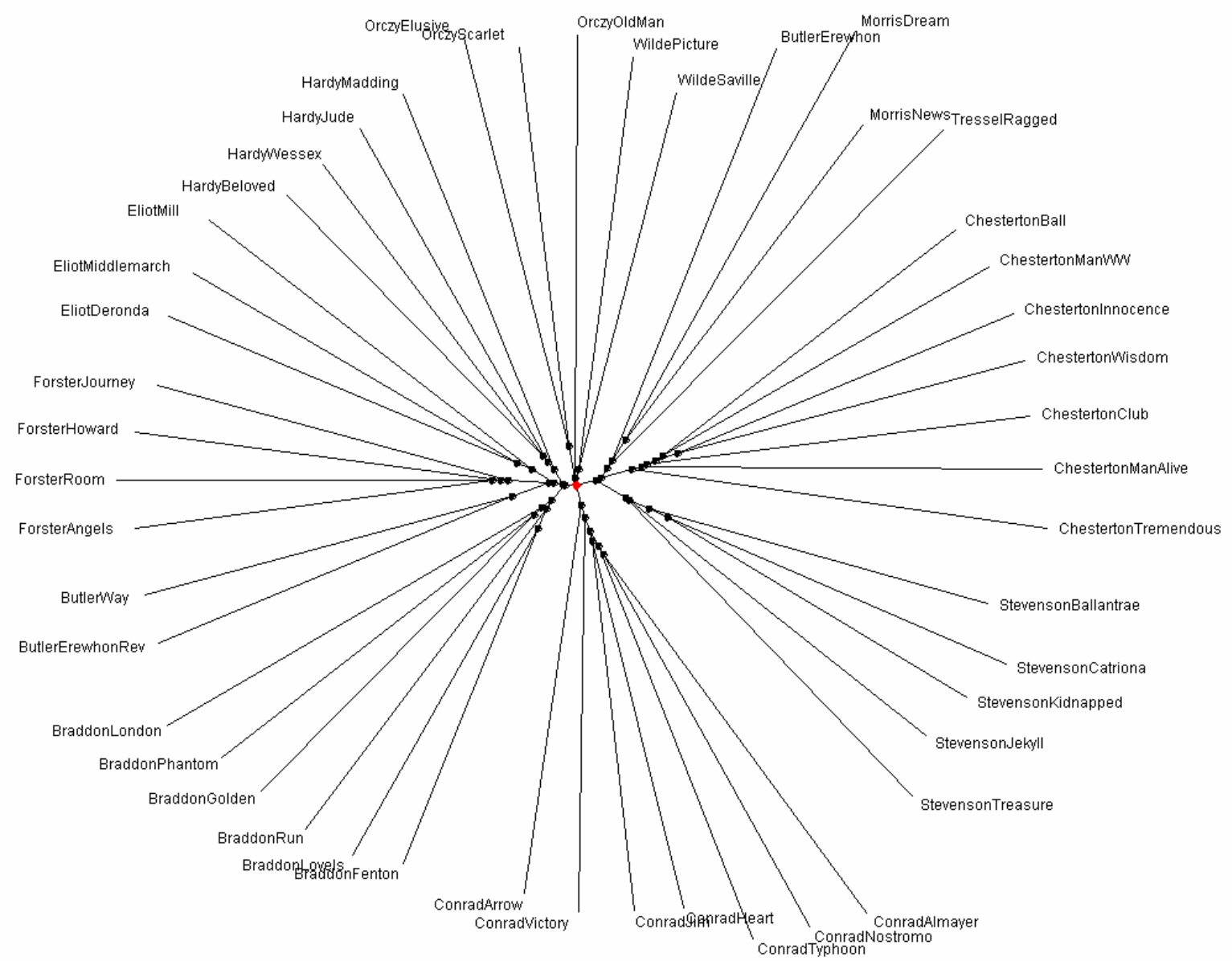

The confidence index for the whole tree is .97 ; for all the nodes, this index is greater than .90 and all the paths are over .80. It is thus possible to believe that the whole tree reproduces, almost faithfully, the information provided by the original matrix of distance indices, but that some of the terminal branches should be considered with care. The "problematic" texts (located in N-NE part of the tree) are also those which are the farthest from the others: Butler's Erewhon, Orczy's Old Man, Tressel's Ragged Philanthropists, or those which are relatively far from their "brothers" (other texts by the same author), and relatively near several others (like Wilde's two novels). None of these problematic texts appears in Table 12 and they are all joined to the root of the tree or very near it. In other words, the algorithm fails to classify them. Thus tree-classification must be improved by excluding these problematic texts from the tree. 
In addition, the "star-shape" must also be noted. As G. Ledger supposed, the vocabularies of the majority of these novels are not very different from each other, even if they are by different authors. One must also admit that the sliding window method erodes, slightly but significantly, the differences between texts (when used on texts the lengths of which greatly differ).

But it must be borne in mind that all these drawbacks were predictable given the fact that the ratio of text lengths in Oxquarry2 was clearly beyond the valid limits of formula (3) and that this experiment was arranged precisely for this reason.

Given these limitations, this method really neutralises the effect of length differences on the distance index as can be observed in Fig. 13 (to be compared with the Fig. 6)

Fig. 13 Correlation between the length of each text and its average distance index with all the others ("sliding window" method: $L=20,000 ; P=2,000$ ).

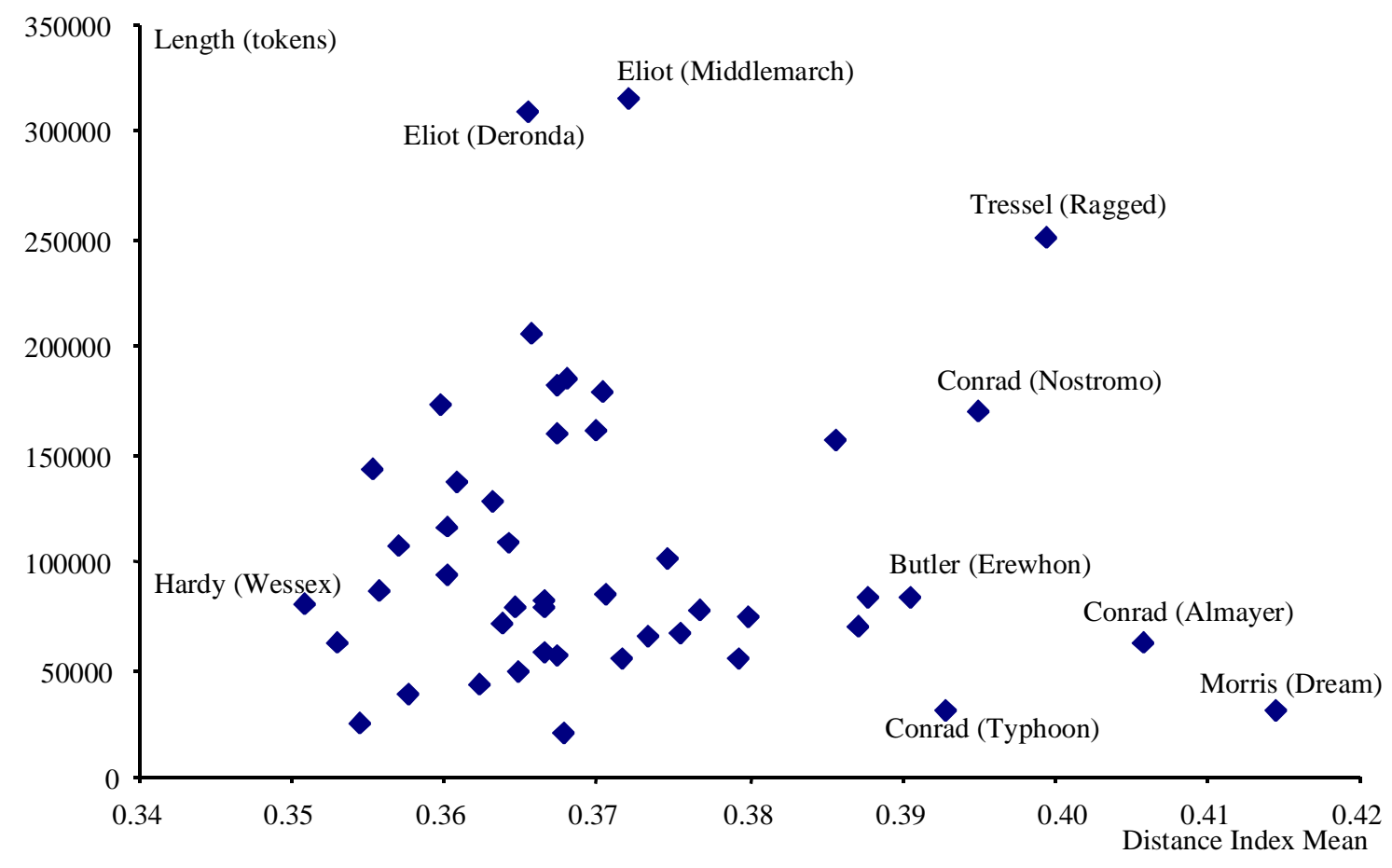

It must also be noted that some books stand apart (on the $\mathrm{X}$-axis): one by Morris (Dream...), the only book of Tressel, three by Conrad (Almayer, Nostromo, Typhoon), and one by Butler (Erewhon). Are these books different from the others because of their topics, of their style? Intertextual distance leads to an interesting question and offers a first possible response with the help of the "contribution to the distance"... 


\section{Conclusions}

A necessary pre-condition must be kept in mind. The spelling of all words must be carefully checked, and a simple rule must be applied to the whole corpus: "one word, one spelling". This standardisation is necessary in order to be sure that the differences are not partly "artificial"...

If texts compared are not too different in size, Formula (3) can be used. When that is not the case, or when more accuracy is needed, the "sliding window" method provides a useful tool. Further experiments carried out on other texts demonstrate that the "sliding window" is not generally sensitive to differences of text size and that its results can be linked to a standardised scale of authorship attribution, like the one presented in the first section of this paper (for $L=10,000$ tokens). In any case, it is preferable not to use these methods when differences of sizes exceed a ratio of 1:8, and to be very cautious when applying them on texts whose lengths are under 10,000 tokens.

Even if the notions of "genre", "topics" and "period" are to be more precisely discussed, these experiments show clearly that, for texts written in the same "genre" by contemporaneous writers, authorship is almost always the dominant factor.

In addition, the sliding window method offers a useful tool with which to analyse "collaborative" texts. When a text is known (or ) suspected to have been written by several hands (e.g. Merriam, 2005), which are the parts by one or other(s) of the potential authors?

Formula (3) and (4) are easy to compute and some interesting characteristics of the two methods may be pointed out:

- Whole texts are considered and, in the calculations, each word type renders exactly its frequency weighting in the text without any "pre-selection" or presupposition about "characteristic" words of each author;

— There is also no prejudging of "sure" or "doubtful" authorship. Given the fact that the method entails no limitation on the number of texts used, it is possible to take into consideration all the available ones (on electronic files), subject to the restrictions of spelling standardisation and of scale ratio cited above.

These two points are important, given the "state of the art" in authorship attribution studies and the numerous critiques which are addressed to them (for example: Rudman, 1998).

Finally, the combination of intertextual distance with modern classification methods considerably furthers the single question of authorship attribution. One is now able to answer some very interesting questions. For example: in a given genre and period, which authors are 
"neighbours" and which are distant? Which are the words which generated this proximity or opposition? Here lies a possible answer to Craig's question: "If you can tell authors apart, have you learned anything about them?" (Craig, 1999)...

\section{Aknowledgments}

The author is grateful to Cyril Labbé (Grenoble I University) who wrote the software with him, to Gerard Ledger for providing the two Corpora Oxquarry and organising the blind-test, and to Thomas Merriam : he had the idea of applying our methods on English texts, he gave me a continuous support and most helpful comments and advice, he carefully edited the first version of this article.

\section{References}

Barthélémy Jean-Pierre and Guénoche Alain (1991). Trees and Proximity Representations, New York, Wiley, 1991).

Burrows John (2002). "“Delta": a Measure of Stylistic Difference and a Guide to Likely Authorship". Literary and Linguistic Computing. 17-3, 267-287.

Burrows John (2003). "Questions of Authorship: Attribution and Beyond". Computers and the Humanities. 37-1, 1-32.

Craig Hugh (1999), "Authorial Attribution and Computational Stylistics: If You Can Tell Authors apart, Have You Learned anything about them?". Literary and Linguistic Computing. 14-1. p 103-113.

Eliott Ward E. Y. and Valenza Robert J. (2001). "Smoking Guns and Silver Bullets: Could John Ford Have Written the Funeral Elegy ?". Literary and Linguistic Computing. 16-3, 205-232.

Hockey Suzan and Martin Jeremy (1988). OCP Users' Manual. Oxford. Oxford University Computing Service.

Holmes David I. (1995). "The Federalist revisited : new directions in autorship attribution". Literary and Linguistic Computing. 10-2. p 111-127.

Hoover David L. (2001). "Statistical Stylistics and Authorship Attribution : an Empirical Investigation". Literary and Linguistic Computing. 16-4. November 2001. p. 421-444.

Labbé Cyril and Labbé Dominique (2001). "Inter-Textual Distance and Authorship Attribution Corneille and Molière". Journal of Quantitative Linguistics. 8-3, 213-231.

Labbé Cyril and Labbé Dominique (2003). "La distance intertextuelle". Corpus. 2, 95-118.

Labbé Cyril and Labbé Dominique (2005). "A tool for Literary Studies. Inter-textual Distance and Tree-Classification". To be published in Literary and Linguistic Computing.

Ledger Gerard R. (1995). "An Exploration of Differences in the Pauline Epistles", Literary and Linguistic Computing. 10-2. 1995. p. 85-97. 
Love Harold (2002). Attributing Authorship: an Introduction. Cambridge. Cambridge University Press.

Luong Xuan (1988). Méthodes d'analyse arborée. Algorithmes, applications. Thèse pour le doctorat ès sciences. Université de Paris V.

Merriam Thomas (2002). "Intertextual Distances between Shakespeare Plays, with Special Reference to Henry V (verse)". Journal of Quantitative Linguistics. 9-3, 260-273.

Merriam Thomas (2003a). "An Application of Authorship Attribution by Intertextual Distance in English". Corpus. 2, p 167-182.

Merriam Thomas (2003b). "Intertextual Distances, Three Authors". Literary and Linguistic Computing, 18-4, 379-388.

Merriam Thomas (2005). The Identity of Shakespeare in Henry VIII. The Renaissance Institute, Tokyo.

Rudman Joseph (1998), "The State of Authorship Attribution Studies: Some Problems and Solutions", Computers and the Humanities, p 351-365.

Rulmann Mathieu, Analyse arborée. Représentation arborée par la méthode des groupements. Grenoble, Polytech'Grenoble et Cerat-IEP, août 2003. 
Appendix 1 "Corpus Oxquarry1" (all excerpt are 10,000 tokens long)

\begin{tabular}{|c|c|c|c|c|c|c|c|}
\hline Except & $\begin{array}{c}\text { Set } 1 \\
\text { Author }\end{array}$ & Titles & Chap. & Except & $\begin{array}{c}\text { Set } 2 \\
\text { Author }\end{array}$ & Titles & Chap. \\
\hline A & Hardy & Jude & I & A & Butler & Erewhon revisit. & XIV \\
\hline B & Butler & Erewhon revisit. & II & B & Morris & Dream of JB & \\
\hline $\mathrm{C}$ & Morris & News & XIII & $\mathrm{C}$ & Tressel & Ragged TP & \\
\hline $\mathrm{D}$ & Stevenson & Catrionae & V & D & Hardy & Jude & \\
\hline $\mathrm{E}$ & Butler & Erewhon revisit. & XVIII & E & Stevenson & Ballantrae & IV \\
\hline $\mathrm{F}$ & Stevenson & Ballantrae & II & $\mathrm{F}$ & Hardy & Wessex Tales & \\
\hline G & Conrad & Lord Jim & XIV & G & Orczy & Elusive P & VII \\
\hline $\mathrm{H}$ & Hardy & Madding & III & $\mathrm{H}$ & Conrad & Lord Jim & XXI \\
\hline I & Orczy & Scarlet P & I & I & Morris & News & VIII \\
\hline $\mathbf{J}$ & Morris & Dream of JB & VII & $\mathrm{J}$ & Hardy & Well beloved & I \\
\hline K & Stevenson & Catrionae & $\mathrm{X}$ & K & Conrad & Almayer & VI \\
\hline $\mathrm{L}$ & Hardy & Jude & VII & $\mathrm{L}$ & Hardy & Well beloved & XII \\
\hline $\mathrm{M}$ & Orczy & Scarlet P & XIV & $\mathrm{M}$ & Morris & News & XIX \\
\hline $\mathrm{N}$ & Stevenson & Ballantrae & $\mathrm{V}$ & $\mathrm{N}$ & Conrad & Almayer & XI \\
\hline $\mathrm{O}$ & Conrad & Lord Jim & VII & $\mathrm{O}$ & Forster & Room with view & I \\
\hline $\mathrm{P}$ & Chesterton & Man who was & I & $\mathrm{P}$ & Forster & Room with view & IV \\
\hline Q & Butler & Erewhon revisit. & VII & Q & Conrad & Almayer & IX \\
\hline $\mathrm{R}$ & Chesterton & Man who was & VII & $\mathrm{R}$ & Stevenson & Catrionae & XVI \\
\hline$S$ & Morris & News & I & S & Hardy & Madding & $\mathrm{X}$ \\
\hline $\mathrm{T}$ & Conrad & Almayer & II & $\mathrm{T}$ & Hardy & Well beloved & $2 \mathrm{VI}$ \\
\hline $\mathrm{U}$ & Orczy & Elusive P & I & $\mathrm{U}$ & Chesterton & Man who was & III \\
\hline V & Conrad & Lord Jim & II & V & Forster & Room with view & VIII \\
\hline W & Orczy & Elusive $\mathrm{P}$ & XIV & W & Stevenson & Catrionae & I \\
\hline $\mathrm{X}$ & Hardy & Wessex Tales & & $X$ & Hardy & Well beloved & VIII \\
\hline $\mathrm{Y}$ & Tressel & Ragged TP & & Y & Orczy & Scarlet P & VII \\
\hline $\mathrm{Z}$ & Tressel & Ragged TP & & $\mathrm{Z}$ & Hardy & Madding & XVIII \\
\hline
\end{tabular}


Appendix 2.1

Lowest intertextual distance indices ranked by ascending values (Corpus Oxquarry1)

\begin{tabular}{|c|c|c|}
\hline Rank & Pair of texts & Index \\
\hline 1 & 1D (StevensonCatri) - 2W (StevensonCatri) & 0.259 \\
\hline 2 & 1N (StevensonBall) - 2E (StevensonBall) & 0.265 \\
\hline 3 & $1 \mathrm{~F}$ (StevensonBall) - 2E (StevensonBall) & 0.267 \\
\hline 4 & 1K (StevensonCatri) - 2W (StevensonCatri) & 0.277 \\
\hline 5 & 1S (MorrisNews) - 2M (MorrisNews) & 0.281 \\
\hline 6 & 2N (ConradAlma) - 2Q (ConradAlma) & 0.283 \\
\hline 7 & 2L (HardyWell) - 2T (HardyWell) & 0.283 \\
\hline 8 & 1C (MorrisNews) - 2I (MorrisNews) & 0.288 \\
\hline 9 & 2J (HardyWell) - 2L (HardyWell) & 0.289 \\
\hline 10 & 1D (StevensonCatri) - 1K (StevensonCatri) & 0.291 \\
\hline 11 & 1E (ButlerErew) - 1Q (ButlerErew) & 0.291 \\
\hline 12 & 2R (StevensonCatri) - 2W (StevensonCatri) & 0.291 \\
\hline 13 & 2J (HardyWell) - 2T (HardyWell) & 0.293 \\
\hline 14 & $1 \mathrm{~F}$ (StevensonBall) - 1N (StevensonBall) & 0.294 \\
\hline 15 & 2I (MorrisNews) - 2M (MorrisNews) & 0.297 \\
\hline 16 & 1D (StevensonCatri) - 2R (StevensonCatri) & 0.302 \\
\hline 17 & $2 \mathrm{O}$ (ForsterRoom) - 2P (ForsterRoom) & 0.302 \\
\hline 18 & 1S (MorrisNews) - 2I (MorrisNews) & 0.303 \\
\hline 19 & 1B (ButlerErew) - 1Q (ButlerErew) & 0.304 \\
\hline 20 & 1Z (TresselRagg) - 2C (TresselRagg) & 0.304 \\
\hline 21 & 2J (HardyWell) - 2X (HardyWell) & 0.306 \\
\hline 22 & 1F (StevensonBall) - 2R (StevensonCatri) & 0.307 \\
\hline 23 & 2E (StevensonBall) - 2R (StevensonCatri) & 0.309 \\
\hline 24 & 1L (HardyJude) - 2J (HardyWell) & 0.310 \\
\hline 25 & 1B (ButlerErew) - 2A (ButlerErew) & 0.310 \\
\hline 26 & 1J (MorrisDream) - 2B (MorrisDream) & 0.311 \\
\hline 27 & 1K (StevensonCatri) - 2R (StevensonCatri) & 0.311 \\
\hline 28 & 1P (ChestertonMan) - 2U (ChestertonMan) & 0.312 \\
\hline 29 & 2L (HardyWell) - 2X (HardyWell) & 0.312 \\
\hline 30 & 2T (HardyWell) - 2X (HardyWell) & 0.313 \\
\hline 31 & 1B (ButlerErew) - 1E (ButlerErew) & 0.314 \\
\hline 32 & 1L (HardyJude) - 2D (HardyJude) & 0.314 \\
\hline 33 & 1L (HardyJude) - 2L (HardyWell) & 0.314 \\
\hline 34 & $1 \mathrm{M}$ (Scar) - 2Y (OrczyScar) & 0.314 \\
\hline 35 & 1X (HardyWess) - 2L (HardyWell) & 0.314 \\
\hline 36 & 1M (Scar) - 1W (OrczyElus) & 0.315 \\
\hline 37 & $2 \mathrm{O}$ (ForsterRoom) - 2V (ForsterRoom) & 0.315 \\
\hline 38 & $1 \mathrm{O}($ ConradLordJ $)$ - 2H (ConradLordJ) & 0.317 \\
\hline 39 & 1P (ChestertonMan) - 1R (ChestertonMan) & 0.317 \\
\hline 40 & $1 \mathrm{G}($ ConradLordJ $)$ - $1 \mathrm{O}$ (ConradLord $)$ & 0.318 \\
\hline 41 & 1H (HardyMadd) - 2Z (HardyMadd) & 0.318 \\
\hline 42 & 1Q (ButlerErew) - 2A (ButlerErew) & 0.318 \\
\hline 43 & 1R (ChestertonMan) - 2U (ChestertonMan) & 0.319 \\
\hline
\end{tabular}




\begin{tabular}{|c|c|c|}
\hline Rank & Pair of texts & Index \\
\hline 44 & 1D (StevensonCatri) - 1N (StevensonBall) & 0.319 \\
\hline 45 & 1D (StevensonCatri) - 2E (StevensonBall) & 0.320 \\
\hline 46 & 1X (HardyWess) - 2J (HardyWell) & 0.320 \\
\hline 47 & 1N (StevensonBall) - 2R (StevensonCatri) & 0.320 \\
\hline 48 & 2F (HardyWess) - 2T (HardyWell) & 0.321 \\
\hline 49 & 2D (HardyJude) - 2J (HardyWell) & 0.322 \\
\hline 50 & 2K (ConradAlma) - 2Q (ConradAlma) & 0.323 \\
\hline 51 & 2D (HardyJude) - 2L (HardyWell) & 0.323 \\
\hline 52 & 1H (HardyMadd) - 2S (HardyMadd) & 0.325 \\
\hline 53 & 1A (HardyJude) - 2D (HardyJude) & 0.325 \\
\hline 54 & 1W (OrczyElus) - 2G (OrczyElus) & 0.325 \\
\hline 55 & 1X (HardyWess) - 2T (HardyWell) & 0.326 \\
\hline 56 & 1F (StevensonBall) - 1K (StevensonCatri) & 0.326 \\
\hline 57 & 2S (HardyMadd) - 2Z (HardyMadd) & 0.326 \\
\hline 58 & 1X (HardyWess) - 2X (HardyWell) & 0.327 \\
\hline 59 & 1K (StevensonCatri) - 1N (StevensonBall) & 0.327 \\
\hline 60 & 2K (ConradAlma) - 2N (ConradAlma) & 0.327 \\
\hline 61 & 1L (HardyJude) - 2T (HardyWell) & 0.329 \\
\hline 62 & 2E (StevensonBall) - 2W (StevensonCatri) & 0.329 \\
\hline 63 & 1E (ButlerErew) - 2A (ButlerErew) & 0.330 \\
\hline 64 & 1L (HardyJude) - 1X (HardyWess) & 0.330 \\
\hline 65 & 2D (HardyJude) - 2T (HardyWell) & 0.330 \\
\hline 66 & 1G (ConradLordJ) - 2H (ConradLordJ) & 0.330 \\
\hline 67 & 1F (StevensonBall) - 2W (StevensonCatri) & 0.330 \\
\hline 68 & 1X (HardyWess) - 2F (HardyWess) & 0.331 \\
\hline 69 & 2F (HardyWess) - 2J (HardyWell) & 0.331 \\
\hline 70 & 1N (StevensonBall) - 2W (StevensonCatri) & 0.332 \\
\hline 71 & 1D (StevensonCatri) - 1F (StevensonBall) & 0.333 \\
\hline 72 & 1L (HardyJude) - 2F (HardyWess) & 0.333 \\
\hline 73 & 1H (HardyMadd) - 2J (HardyWell) & 0.335 \\
\hline 74 & 2D (HardyJude) - 2X (HardyWell) & 0.335 \\
\hline 75 & 1Y (TresselRagg) - 1Z (TresselRagg) & 0.337 \\
\hline 76 & 1H (HardyMadd) - 2T (HardyWell) & 0.337 \\
\hline 77 & 2F (HardyWess) - 2L (HardyWell) & 0.338 \\
\hline 78 & 1K (StevensonCatri) - 2E (StevensonBall) & 0.338 \\
\hline 79 & 2P (ForsterRoom) - 2V (ForsterRoom) & 0.338 \\
\hline 80 & 1X (HardyWess) - 2D (HardyJude) & 0.339 \\
\hline 81 & 1H (HardyMadd) - 2D (HardyJude) & 0.340 \\
\hline 82 & 1H (HardyMadd) - 2L (HardyWell) & 0.340 \\
\hline 83 & 1H (HardyMadd) - 1L (HardyJude) & 0.344 \\
\hline 84 & 1L (HardyJude) - 2X (HardyWell) & 0.344 \\
\hline 85 & 1W (OrczyElus) - 2Y (OrczyScar) & 0.344 \\
\hline 86 & 1K (StevensonCatri) - 1S (MorrisNews) & 0.349 \\
\hline
\end{tabular}


Appendix 2.2

Pairs of texts of which the inter-textual distance indices are between .35 and .36 (bold types : same authorship)

\begin{tabular}{|c|c|c|}
\hline Rank & Pairs of texts & Indices \\
\hline 87 & 1S (MorrisNews) - 2W (StevensonCatri) & 0.350 \\
\hline 88 & 1A (HardyJude) - 1L (HardyJude) & $\mathbf{0 . 3 5 0}$ \\
\hline 89 & 2G (OrczyElus) - 2Y (OrczyScar) & $\mathbf{0 . 3 5 0}$ \\
\hline 90 & 1A (HardyJude) - 2J (Hardy Well) & 0.350 \\
\hline 91 & 1Y (TresselRagg) - 2C (TresselRagg) & 0.351 \\
\hline 92 & 1F (StevensonBall) - 1G (ConradLordJ) & 0.351 \\
\hline 93 & 1H (HardyMadd) - 2F (HardyWess) & 0.351 \\
\hline 94 & 1C (MorrisNews) - 2M (MorrisNews) & 0.351 \\
\hline 95 & 2J (HardyWell) - 2Z (HardyMadd) & 0.351 \\
\hline 96 & 1M (OrczyScar) - 2G (OrczyElus) & 0.352 \\
\hline 97 & 1A (HardyJude) - 1H (HardyMadd) & 0.353 \\
\hline 98 & 1C (MorrisNews) - 1S (MorrisNews) & 0.353 \\
\hline 99 & 1L (HardyJude) - 1Z (TresselRagg) & 0.353 \\
\hline 100 & 1L (HardyJude) - 2S (HardyMadd) & 0.354 \\
\hline 101 & 2F (HardyWess) - 2X (HardyWell) & 0.354 \\
\hline 102 & 1A (HardyJude) - 2T (HardyWell) & 0.355 \\
\hline 103 & 1Q (ButlerErew) - 2J (HardyWell) & 0.355 \\
\hline 104 & 2T (HardyWell) - 2Z (HardyMadd) & 0.355 \\
\hline 105 & 1H (HardyMadd) - 1X (HardyWess) & 0.355 \\
\hline 106 & $1 \mathrm{G}($ ConradLordJ) - 1N (StevensonBall) & 0.356 \\
\hline 107 & 1H (HardyMadd) - 2X (Hardy Well) & 0.357 \\
\hline 108 & 2J (HardyWell) - 2S (HardyMadd) & 0.357 \\
\hline 109 & 1G (ConradLordJ) - 2E (StevensonBall) & 0.357 \\
\hline 110 & $10($ ConradLordJ $)$ - 1V $($ ConradLordJ $)$ & 0.357 \\
\hline 111 & 1Q (ButlerErew) - 2L (HardyWell) & 0.357 \\
\hline 112 & 1S (MorrisNews) - 2R (StevensonCatri) & 0.357 \\
\hline 113 & 1T (ConradAlma) - 2K (ConradAlma) & 0.357 \\
\hline 114 & 1F (StevensonBall) - 1S (MorrisNews) & 0.357 \\
\hline 115 & 2D (HardyJude) - 2Z (HardyMadd) & 0.358 \\
\hline 116 & 1F (StevensonBall) - 2H (ConradLordJ) & 0.358 \\
\hline 117 & 1A (HardyJude) - 2L (HardyWell) & 0.358 \\
\hline 118 & 2D (HardyJude) - 2H (ConradLordJ) & 0.359 \\
\hline 119 & 1A (HardyJude) - 1X (HardyWess) & 0.359 \\
\hline 120 & 2H (ConradLordJ) - 2X (HardyWell) & 0.359 \\
\hline 121 & 1X (HardyWess) - 2Z (HardyMadd) & 0.359 \\
\hline 122 & 2D (HardyJude) - 2F (HardyWess) & 0.359 \\
\hline 123 & 2D (HardyJude) - 2S (HardyMadd) & 0.360 \\
\hline 124 & 2F (HardyWess) - 2S (HardyMadd) & 0.360 \\
\hline 125 & 1L (HardyJude) - 1Q (ButlerErew) & 0.360 \\
\hline 126 & 1A (HardyJude) - 2X (HardyWell) & 0.360 \\
\hline 127 & 2L (HardyWell) - 2Z (HardyMadd) & 0.360 \\
\hline
\end{tabular}


Appendix 2.3

Pairs of excerpts by a same author separated by intertextual distance indices over .36

\begin{tabular}{|c|c|c|}
\hline Rank & Pairs of texts & Indices \\
\hline 134 & 2F (HardyWess) - 2Z (HardyMadd) & 0.363 \\
\hline 139 & 1I (OrczyScar) - 2Y (OrczyScar) & 0.364 \\
\hline 143 & 2L (HardyWell) - 2S (HardyMadd) & 0.365 \\
\hline 144 & 1L (HardyJude) - 2Z (HardyMadd) & 0.365 \\
\hline 145 & 2S (HardyMadd) - 2T (HardyWell) & 0.365 \\
\hline 151 & 1X (HardyWess) - 2S (HardyMadd) & 0.366 \\
\hline 153 & $1 \mathrm{G}($ ConradLordJ $)-1 \mathrm{~V}($ ConradLordJ $)$ & 0.367 \\
\hline 157 & 1A (HardyJude) - 2F (HardyWess) & 0.367 \\
\hline 171 & 1U (OrczyElus) - 2Y (OrczyScar) & 0.370 \\
\hline 172 & 2X (HardyWell) - 2Z (HardyMadd) & 0.370 \\
\hline 179 & 1J (MorrisDream) - 2I (MorrisNews) & 0.371 \\
\hline 186 & $1 \mathrm{~V}$ (ConradLordJ) - 2H (ConradLordJ) & 0.373 \\
\hline 187 & 1F (StevensonBall) - 1R (ChestertonManW) & 0.373 \\
\hline 209 & 1U (OrczyElus) - 1W (OrczyElus) & 0.376 \\
\hline 213 & 1A (HardyJude) - 2Z (HardyMadd) & 0.376 \\
\hline 224 & 1A (HardyJude) - 2S (HardyMadd) & 0.378 \\
\hline 229 & 1S (MorrisNews) - 2B (MorrisDream) & 0.378 \\
\hline 233 & 1C (MorrisNews) - 1J (MorrisDream) & 0.379 \\
\hline 235 & 1V (ConradLordJ) - 2N (ConradAlma) & 0.379 \\
\hline 253 & 1J (MorrisDream) - 2M (MorrisNews) & 0.380 \\
\hline 256 & 1I (OrczyScar) - 1U (OrczyElus) & 0.381 \\
\hline 261 & 1J (MorrisDream) - 1S (MorrisNews) & 0.381 \\
\hline 265 & 1U (OrczyElus) - 2G (OrczyElus) & 0.381 \\
\hline 274 & 2H (ConradLordJ) - 2N (ConradAlma) & 0.382 \\
\hline 305 & 1T (ConradAlma) - 2Q (ConradAlma) & 0.384 \\
\hline 307 & $1 \mathrm{O}$ (ConradLordJ) - 2N (ConradAlma) & 0.385 \\
\hline 343 & $1 \mathrm{O}$ (ConradLordJ) - 2Q (ConradAlma) & 0.387 \\
\hline 365 & 1V (ConradLordJ) - 2K (ConradAlma) & 0.389 \\
\hline 408 & 2S (HardyMadd) - 2X (HardyWell) & 0.391 \\
\hline 420 & 1T (ConradAlma) - 2N (ConradAlma) & 0.392 \\
\hline 463 & 1M (OrczyScar) - 1U (OrczyElus) & 0.395 \\
\hline
\end{tabular}

The remaining 863 indices (over .395) are all between excerpts by different authors 
Appendix 2.4

Pairs of excerpts separated by intertextual distance indices over .48

\begin{tabular}{|c|c|c|}
\hline 1301 & 1N (StevensonBall) - 1T (ConradAlma) & 0.481 \\
\hline 1302 & 2K (ConradAlma) - 2V (ForsterRoom) & 0.483 \\
\hline 1303 & 1D (StevensonCatri) - 1V (ConradLordJ) & 0.483 \\
\hline 1304 & 1T (ConradAlma) - 1Z (TresselRagg) & 0.484 \\
\hline 1305 & 1T (ConradAlma) - 2R (StevensonCatri) & 0.486 \\
\hline 1306 & 2K (ConradAlma) - 2W (StevensonCatri) & 0.486 \\
\hline 1307 & 1D (StevensonCatri) - 2K (ConradAlma) & 0.486 \\
\hline 1308 & 1J (MorrisDream) - 2K (ConradAlma) & 0.488 \\
\hline 1309 & $1 \mathrm{~J}$ (MorrisDream) - 1V (ConradLordJ) & 0.489 \\
\hline 1310 & 1T (ConradAlma) - 2M (MorrisNews) & 0.490 \\
\hline 1311 & 1T (ConradAlma) - $2 \mathrm{O}$ (ForsterRoom) & 0.490 \\
\hline 1312 & 1C (MorrisNews) - 1T (ConradAlma) & 0.491 \\
\hline 1313 & 1T (ConradAlma) - 2A (ButlerErew) & 0.491 \\
\hline 1314 & 1T (ConradAlma) - 2B (MorrisDream) & 0.492 \\
\hline 1315 & 1T (ConradAlma) - 2C (TresselRagg) & 0.494 \\
\hline 1316 & 1T (ConradAlma) - 2I (MorrisNews) & 0.496 \\
\hline 1317 & 1S (MorrisNews) - 1T (ConradAlma) & 0.496 \\
\hline 1318 & 1B (ButlerErew) - 1T (ConradAlma) & 0.497 \\
\hline 1319 & 1Q (ButlerErew) - 1T (ConradAlma) & 0.499 \\
\hline 1320 & $1 \mathrm{~T}$ (ConradAlma) - 2V (ForsterRoom) & 0.500 \\
\hline 1321 & 1J (MorrisDream) - 2G (OrczyElus) & 0.503 \\
\hline 1322 & 1E (ButlerErew) - 1T (ConradAlma) & 0.508 \\
\hline 1323 & 1K (StevensonCatri) - 1T (ConradAlma) & 0.508 \\
\hline 1324 & 1D (StevensonCatri) - 1T (ConradAlma) & 0.511 \\
\hline 1325 & 1T (ConradAlma) - 2W (StevensonCatri) & 0.513 \\
\hline 1326 & $1 \mathrm{~J}$ (MorrisDream) - 1T (ConradAlma) & 0.525 \\
\hline
\end{tabular}


Appendix 3. Corpus Oxquarry 2

\begin{tabular}{|c|c|c|c|}
\hline Author & Title & $\begin{array}{c}\text { Length } \\
\text { (tokens) }\end{array}$ & $\begin{array}{c}\text { Vocabulary } \\
\text { (types) }\end{array}$ \\
\hline Braddon Mary & Fenton's Quest & 172,952 & 10,154 \\
\hline Braddon Mary & The Golden Calf & 160,172 & 12,490 \\
\hline Braddon Mary & London Pride & 157,238 & 13,498 \\
\hline Braddon Mary & The Lovels of Arden & 182,428 & 11,832 \\
\hline Braddon Mary & Phantom Fortune & 178,994 & 12,886 \\
\hline Braddon Mary & Run to Earth & 185,627 & 10,635 \\
\hline Butler Samuel & Erewhon & 83,905 & 7,886 \\
\hline Butler Samuel & Erewhon Revisited & 84,853 & 6,706 \\
\hline Butler Samuel & The Way of all Flesh & 161,847 & 10,611 \\
\hline Chesterton G. K. & The Bell and the Cross & 79,445 & 8,126 \\
\hline Chesterton G. K. & The Club of Queer Trades & 44,017 & 5,663 \\
\hline Chesterton G. K. & The Innocence of Father Brown & 78,902 & 8,169 \\
\hline Chesterton G. K. & Man Alive & 57,553 & 7,492 \\
\hline Chesterton G. K. & The Man who was Thursday & 58,066 & 6,494 \\
\hline Chesterton G. K. & Tremendous Trifles & 55,969 & 6,821 \\
\hline Chesterton G. K. & The Wisdom of Father Brown & 71,588 & 8,124 \\
\hline Conrad Joseph & Almayer's folly & 62,575 & 6,483 \\
\hline Conrad Joseph & The Arrow of Gold & 107,193 & 8,663 \\
\hline Conrad Joseph & Heart of Darkness & 20,243 & 4,021 \\
\hline Conrad Joseph & Lord Jim & 128,685 & 10,970 \\
\hline Conrad Joseph & Nostromo & 170,103 & 12,805 \\
\hline Conrad Joseph & Typhoon & 31,024 & 5,009 \\
\hline Conrad Joseph & Victory & 117,319 & 9,408 \\
\hline Eliot George & Daniel Deronda & 309,013 & 15,829 \\
\hline Eliot George & The Mill on the Floss & 206,830 & 12,805 \\
\hline Eliot George & Middlemarch & 316,156 & 16,369 \\
\hline Forster E. M. & Where Angels Fear to Tread & 49,449 & 5,636 \\
\hline Forster E. M. & Howard's End & 108,546 & 9,367 \\
\hline Forster E. M. & The Longest Journey & 93,986 & 8,478 \\
\hline Forster E. M. & A Room with a View & 66,456 & 6,920 \\
\hline Hardy Thomas & Beloved & 62,839 & 7,191 \\
\hline Hardy Thomas & Jude & 143,996 & 11,294 \\
\hline Hardy Thomas & Far from the madding crowd & 137,879 & 11,958 \\
\hline Hardy Thomas & Wessex tales & 80,694 & 8,231 \\
\hline Morris William & Dream & 31,398 & 3,661 \\
\hline Morris William & News from nowhere & 77,721 & 6,682 \\
\hline Orczy Baroness E. & The Elusive Pimpernel & 83,314 & 8,114 \\
\hline Orczy Baroness E. & The Old Man in the Corner & 69,591 & 6,365 \\
\hline Orczy Baroness E. & League of the Scarlet Pimpernel & 75,208 & 7,151 \\
\hline Stevenson Robert L. & Ballantrae & 87,156 & 7,694 \\
\hline Stevenson Robert L. & Catronia & 101,012 & 7,940 \\
\hline Stevenson Robert L. & Doctor Jekyll & 25,646 & 4,012 \\
\hline Stevenson Robert L. & Kidnapped & 81,706 & 6,829 \\
\hline Stevenson Robert L. & Treasure Island & 67,883 & 6,143 \\
\hline Tressel Robert & The Ragged Trousered Philanthropists & 251,159 & 12,423 \\
\hline Wilde Oscar & The Picture of Dorian Gray & 54,604 & 5,612 \\
\hline Wilde Oscar & Lord Arthur Savilles Crimes & 39,311 & 5,351 \\
\hline
\end{tabular}

